\title{
Análise biogeográfica da avifauna da região oeste do baixo Rio Negro, amazônia brasileira
}

\author{
Sérgio H. Borges \\ Fundação Vitória Amazônica. Rua Estrela D’Alva, casa 7, Conjunto Morada do Sol, Aleixo, 69060-510 Manaus, Amazonas. \\ E-mail: sergio@fva.org.br
}

\begin{abstract}
Biogeographical analysis of the avifauna in the lower western Rio Negro region in the Brazilian Amazon. This study makes a detailed biogeographical analysis of Central Amazon avifauna west of the Rio Negro, where Parque Nacional do Jaú (PN]) is located. The geographical distribution of 383 bird taxa (species or subspecies) recorded in PN] was analyzed through qualitative and quantitative biogeographic methods (cluster analysis and parsimony analysis of endemicity). Results showed that the avifauna of PN] is strongly influenced by the northwestern and western regions of the Amazon Basin. Three biogeographic units were identified and PN] grouped with sites in the upper Orinoco river and Guiana. Similarly, JNP grouped with the upper Orinoco river and Guiana is the sister-group in the area cladogram with strongest support. Different subgroups of species reveal area cladograms with different topologies, suggesting complex scenarios of avifauna diversification. These scenarios may have been influenced by geological events occurring during the Miocene and Pleistocene. In this study was identified an area of endemism previously not described for the Amazon, the Rio Negro endemic area. The avifauna of PN] is representative of the northwestern and western Amazon and has taxa with distributions restricted to the central region of the Amazon, reinforcing its importance in protecting the biodiversity of the Amazon.

KEY WORDS. Amazon biogeography; areas of endemism; Miocene; Neotropical birds; parsimony analysis of endemicity; Pleistocene.
\end{abstract}

RESUMO. Neste estudo é realizada uma análise biogeográfica detalhada da avifauna da Amazônia Central a oeste do Rio Negro, região onde está localizado o Parque Nacional do Jaú (PNJ). As distribuições geográficas de 383 táxons (espécies e subespécies) de aves registradas no PN] foram analisadas através de métodos biogeográficos qualitativos e quantitativos (análise de agrupamento e análise de parcimônia de endemismo). A avifauna do PN] é fortemente influenciada pelas regiões noroeste e oeste da bacia amazônica. Foram identificadas três unidades biogeográficas e o PN] se agrupa com sítios localizados no alto Rio Orinoco e na Guiana. De modo similar, o PN] se agrupa com o sítio do alto Rio Orinoco tendo a Guiana como grupo-irmão no cladograma de área com maior suporte. Diferentes sub-grupos de espécies revelaram cladogramas de áreas com variadas topologias, sugerindo complexos cenários de diversificação da avifauna amazônica. Estes cenários biogeográficos podem ter sido influenciados por eventos geológicos ocorridos durante o Mioceno e Pleistoceno. Neste estudo foi identificada uma área de endemismo não descrita para a Amazônia - a área de endemismo Rio Negro. A avifauna da região do PN], além de ser representativa dos setores noroeste e oeste da Amazônia, possui táxons de distribuição restrita à região central da Amazônia, reforçando ainda mais sua importância na proteção da biodiversidade amazônica.

PALAVRAS-CHAVE. Aves neotropicais; análise de parcimônia de endemismo; áreas de endemismos; biogeografia da Amazônia; Mioceno; Pleistoceno.

Hipóteses sobre como as espécies evoluíram no tempo e no espaço são construídas e testadas a partir da interação de fases distintas e complementares de estudos biogeográficos que incluem a construção de uma sólida base de dados sobre a distribuição das espécies, a identificação de padrões de distribuição, a delimitação de áreas de endemismo de espécies que comparti- lham histórias evolutivas comuns, o estabelecimento de interrelações entre estas áreas de endemismo e a identificação de processos físicos e biológicos que podem atuar como mecanismos causais destas distribuições (Ball 1975, Cracraft 1983, Rosen 1995a). O desenvolvimento dos estudos biogeográficos sobre as aves amazônicas seguiram, em linhas gerais, estas fases. 
Naturalistas nos séculos passados e ornitólogos modernos vêm continuamente acumulando dados sobre a distribuição geográfica das aves amazônicas. Pesquisadores também começaram a notar padrões recorrentes na distribuição de espécies de aves na Amazônia. Um dos padrões melhor conhecido é a influência do sistema hidrográfico sobre a distribuição das aves, onde várias espécies aparentadas (mesmo gênero ou diferentes subespécies) se substituem em margens opostas dos grandes tributários da bacia amazônica como os rios Negro, Madeira e o próprio Solimões/Amazonas (Sick 1967, Haffer 1992).

Outros padrões de distribuição de aves amazônicas também começaram a ser identificados a partir dos trabalhos do ornitólogo alemão Jürgen Haffer. Haffer reuniu e analisou uma enorme quantidade de dados de coletas de aves identificando as grandes unidades biogeográficas da América do Sul baseadas na distribuição das aves (Haffer 1969, 1974). Os estudos de Haffer foram ampliados por CRACRAFT (1985), que identificou 33 áreas de endemismo para a avifauna da América do Sul.

Além de auxiliarem na delimitação de unidades biogeográficas, ornitólogos e outros especialistas propuseram vários mecanismos de especiação para explicar a grande diversidade de espécies da biota amazônica. Entre estas hipóteses biogeográficas estão aqueles que destacam a importância dos gradientes ecológicos na geração de espécies simpátricas (ENDLER 1982, SMITH et al. 1997), a suposta existência de refúgios florestais pleistocênicos (HAFFER 1969), a influência do sistema hidrográfico na diversificação da biota (Ayres \& Clutton-Brock 1992, HAFFer 1992), os arcos geológicos espalhados pela bacia amazônica como indicadores de processos de vicariância (Silva \& PatTon 1998, PATTON \& Silva 2001) e o papel biogeográfico dos mares e lagos salobros que invadiram a bacia amazônica (MARROIg \& CERQUEIRA 1997, NoREs 1999, BATEs 2001). Estas hipóteses biogeográficas são complexas e descrições mais completas podem ser encontradas em Haffer (2001) e Colinvaux et al. (2001).

Análises biogeográficas da distribuição de aves na Amazônia são importantes por ajudarem na delimitação de áreas de endemismos, na geração de hipóteses sobre mecanismos causais de especiação e na identificação de áreas relevantes para a conservação da biodiversidade (SICK 1967, HAFFER 1969, 1974, Wetteberg et al. 1976, Cracraft 1983, 1985, Rylands 1991, Bates et al. 1998). A relativa escassez de conhecimento sobre a distribuição das espécies de aves em alguns setores da bacia, entretanto, têm dificultados estas aplicações. Mesmo locais considerados como bem amostrados ainda podem revelar importantes registros ornitológicos (Oren \& Albuquerque 1991, Aleixo et al. 2000). Estudos mais regionalizados, portanto, podem revelar aspectos importantes e desconhecidos da biogeografia de aves na bacia amazônica.

A região oeste do baixo Rio Negro foi apontada como uma área de grande relevância para estudos ornitológicos (OREN \& Albuquerque 1991). Os trabalhos de campo nesta região iniciaram-se com os inventários intensivos e estudos ecológicos sobre a aves do Parque Nacional do Jaú (Borges \& Carvalhaes 2000,
Borges et al. 2001, Borges 2004). O Parque Nacional do Jaú (PNJ) está localizado entre os rios Negro, Japurá e Solimões, uma posição intermediária entre as áreas de endemismos já mapeadas para aves amazônicas (HafFer 1974, 1978, Cracraft 1985, Silva et al. 2002). A região também é reconhecida como uma área de contato secundário entre espécies que, hipoteticamente, se originaram na periferia da bacia amazônica (HAFFer 1985).

No presente artigo, é apresentado um estudo biogeográfico detalhado da avifauna do setor oeste do Rio Negro através de uma análise da distribuição geográfica das aves registradas no PNJ. As principais questões que nortearam o estudo foram: Quais são as afinidades biogeográficas entre o setor oeste do baixo Rio Negro e outras regiões da Amazônia? Quais espécies de aves caracterizam a posição biogeográfica da região do PNJ e como estes elementos se distribuem ecologicamente? Qual a relação biogeográfica entre a região do Parque e as áreas de endemismos de aves amazônicas? A região central da Amazônia, em sua porção oeste do Rio Negro, é uma região de transição entre áreas de endemismos ou existem espécies restritas a este setor?

\section{MATERIAL E MÉTODOS}

\section{Área de estudo}

O Parque Nacional do Jaú (PNJ) é umas das maiores unidades de conservação do bioma amazônico, ocupando uma área de 2.272.000 hectares localizados entre os rios Negro, Solimões e Japurá (Fig. 1). A região do Parque incorpora um mosaico de vários tipos de vegetação florestal e não florestal classificadas em: matas de terra firme, matas inundadas por rios de água preta ou matas de igapó, vegetações de campinas e campinaranas associadas a solos arenosos e outros tipos de vegetação com distribuições mais restritas como capoeiras e vegetações sobre solos hidromórficos dos setores interfluviais do Parque (FERREIRA 1997, Ferreira \& Prance 1998, Borges et al. 2001, Vicentini 2004). A avifauna de vários destes tipos de vegetação foi alvo de estudos específicos permitindo um bom entendimento do uso de hábitat pelas aves do PNJ (Borges \& Carvalhaes 2000, Borges 2004). Informações mais detalhadas sobre o PNJ incluindo descrições de vegetação, clima, solos e geologia podem ser obtidas em (FerreirA 1997, Ferreira \& Prance 1998, Fva/ibama 1998, Borges et al. 2001, VicENTINI 2004).

\section{Distribuição geográfica e taxonomia}

Este estudo é baseado em uma análise detalhada da listagem publicada de aves do PNJ, atualizada em trabalhos de campo mais recentes (Borges et al. 2001). Aves aquáticas (e.g. Ardeidae e Scolopacidae), migrantes (e.g. Hirundinidae, Tyrannus savana Vieillot, 1808 - Tyrannidae) e espécies cujas identificações não foram conclusivas foram excluídas das análises, o que corresponde a cerca de 18,5\% das espécies registradas no PNJ. Todas as espécies politípicas de aves foram identificadas em nível de subespécie utilizando informações das seguintes fontes: a) coleta de espécimens na região: aproximadamente 114 espécies de aves que ocorrem no PNJ estão representadas por 


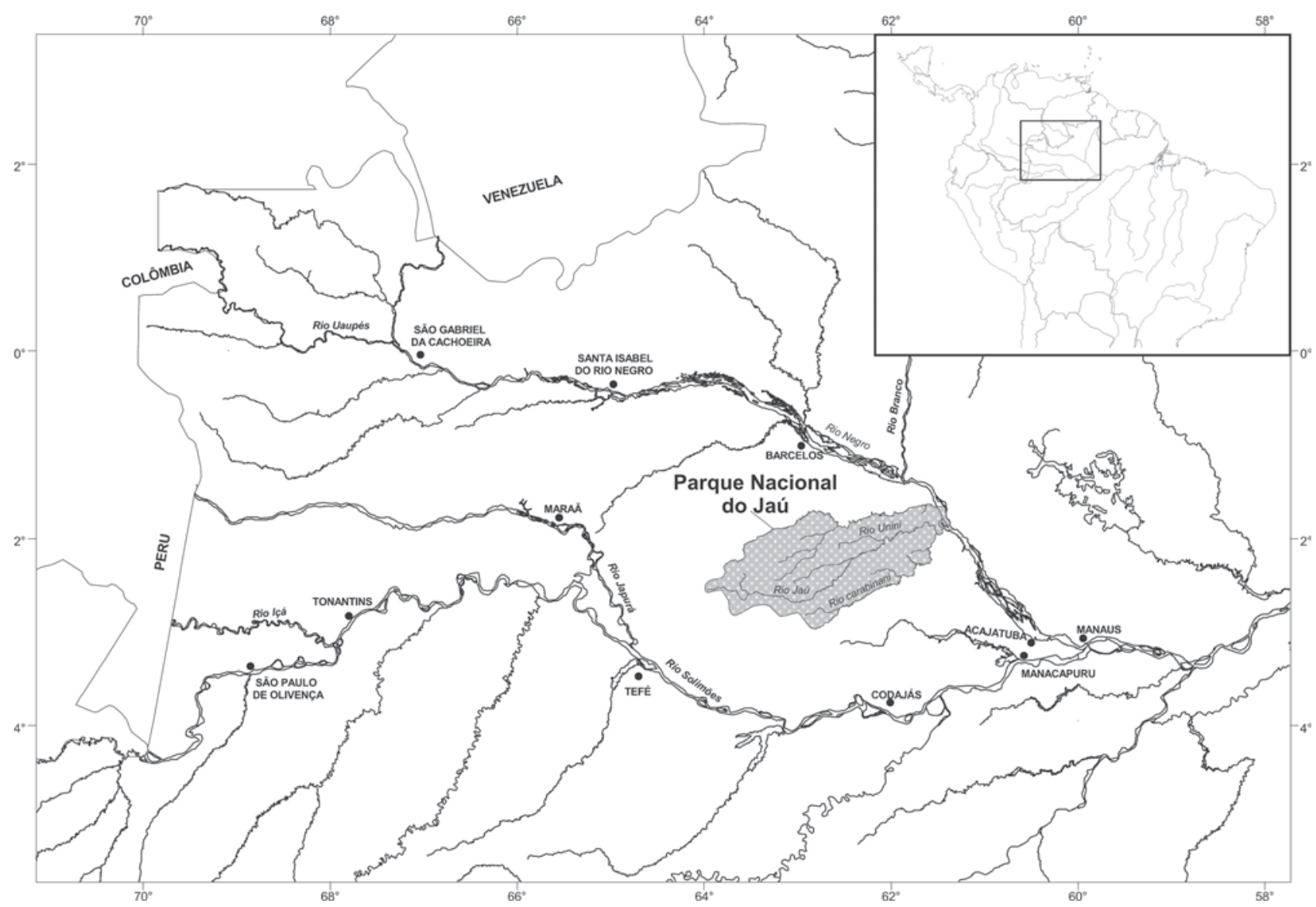

Figura 1. Mapa do noroeste da Amazônia com a localização do Parque Nacional do Jaú. Os pontos se referem às sedes dos munícipios citados no texto.

espécimens coletados e depositados na coleção de aves do Museu Paraense Emílio Goeldi (MPEG). Comparações deste material com exemplares já identificados na coleção do MPEG e descrição de subespécies na literatura especializada permitiram identificações seguras destes exemplares; b) análise de espécimens coletados no interflúvio Negro/Japurá: o MPEG possui uma coleção de aves coletadas nas proximidades do município de Maraã localizado na margem esquerda do Rio Japurá. Além disto, existem vários exemplares coletados em Barcelos e Moura, municípios localizados a cerca de 50 quilômetros ao norte da foz do Rio Jaú (Novaes 1994). Estes municípios estão localizados no mesmo interflúvio que o PNJ (Fig. 1), sendo razoável admitir que as subespécies coletadas nestes setores são as mesmas que ocorrem no Parque. De fato, comparações entre exemplares coletados em Maraã, Barcelos, Moura e PNJ indicam que estas subespécies devem se distribuir de modo contínuo pelo interflúvio Negro/Japurá. Foram analisados exemplares de 295 espécies ou subespécies de aves coletadas no interflúvio $\mathrm{Ne}$ gro/Japurá representando $77 \%$ dos taxóns analisados no presente estudo; c) identificação por proximidade geográfica: as dis- tribuições geográficas das espécies de aves registradas no PNJ que não possuem exemplares coletados, foram estudadas utilizando como referências principais os catálogos de PINTo (1944, 1978), Friedmann (1948) e outras obras de referência (Ridgely \& Tudor 1989, 1994, del Hoyo et al. 1992, 1993, 1996, 1997, 1999, 2001, 2002, 2003, 2004). Nestes casos, a identificação das subespécies foi feita por proximidade geográfica com o setor oeste do Rio Negro.

A taxonomia e a distribuição geográfica dos táxons de aves adotadas no presente estudo segue Pinto (1944, 1978), NorgaArden-Olesen $(1973,1974)$, Isler \& Isler (1987), Del Hoyo et al. (1992, 1993, 1996, 1997, 1999, 2001, 2002, 2003, 2004), Ridgely \& Tudor $(1989,1994)$ e revisões taxonômicas mais recentes de espécies ou grupos de espécies (ver notas taxonômicas e biogeográficas no Anexo I).

\section{Biogeografia descritiva}

As distribuições das espécies e subespécies de aves do PNJ foram categorizadas seguindo uma divisão da bacia amazônica em quatro setores como sugerido por WALLACE (1852): os seto- 
res leste e oeste do Rio Negro e os setores leste e oeste do Rio Madeira. Esta divisão biogeográfica se justifica pelo fato de que os limites de distribuição de várias espécies e subespécies de aves na Amazônia coincidem com os grandes rios da bacia, principalmente o Madeira, Amazonas e Negro (HAFFer 1992). As distribuições geográficas das aves do PNJ foram classificadas em: (a) espécies de distribuição ampla pela Amazônia que ocorrem em três ou mais dos setores definidos acima; (b) espécies restritas ao noroeste da Amazônia no sul da Venezuela, noroeste do Brasil e sudeste da Colômbia. Uma boa parte destas espécies só ocorre na margem direita do Rio Negro. As distribuições de algumas espécies classificadas nesta categoria se estendem até Roraima e Guianas, mas não ocorrem na Amazônia Central a leste do Rio Negro (e.g. Manaus); (c) espécies de distribuição ampla ao norte do Rio Solimões/Amazonas incluindo ambas as margens do Rio Negro (e.g. Microbates collaris collaris Pelzeln, 1868 - Sylviidae; (d) espécies que se distribuem no noroeste e oeste da Amazônia em ambas as margens do Rio Solimões/ Amazonas; (e) espécies que se distribuem no noroeste e oeste da Amazônia, mas são restritas ao norte do Rio Solimões/Amazonas; (f) espécies do oeste da Amazônia em ambas as margens do Solimões/Amazonas, mas aparentemente não registradas na porção noroeste da bacia; (g) espécies restritas ao centro-oeste da Amazônia entre os rios Negro e Solimões; (h) espécies registradas no oeste da Amazônia ao norte do Rio Solimões/ Amazonas, mas não encontradas no noroeste da bacia; (i) espécies com outros tipos de distribuição: neste caso são incluídas espécies que não se enquadram nas grandes categorias geográficas definidas acima. Um exemplo de ave desta categoria é o pequeno passeriforme Myrmotherula klagesi Todd, 1927 Thamnophilidae, encontrada na região do médio Amazonas (Santarém) e baixo Rio Negro (Ridgely \& Tudor 1994).

\section{Biogeografia quantitativa}

As análises biogeográficas quantitativas foram baseadas nas comparações entre as listagens de espécies de aves das seguintes regiões da Amazônia (Fig. 2): 1) Quebrada Sucusari, Peru, a oeste do Rio Napo. Uma listagem de aves desta região foi gentilmente cedida pelo ornitólogo Bret Whitney (B. Whitney, Museum of Natural Science - Louisiana State University, com. pess.), 2) região do alto Rio Orinoco na Venezuela (Zimmer \& Hilty 1997), 3) Parque Nacional do Jaú (presente estudo), 4) região norte de Manaus (CoHn-Haft et al. 1997), 5) região de Belém e proximidades (Novaes \& Lima 1998), 6) Alta Floresta no norte do Mato Grosso (Zimmer et al. 1997), 7) Cachoeira de Nazaré na região oeste de Rondônia (Stotz et al. 1997) e 8) Rio Urucu no oeste da Amazônia (Peres \& Whittaker 1991). Comparações entre listagens de espécies de aves para diferentes propostas apresentam problemas intrínsecos incluindo técnicas utilizadas nos estudos, habilidade dos ornitólogos em identificar as espécies, esforço de amostragem e hábitats estudados (REMSEN 1994). Foram adotados alguns critérios para tornar as listagens mais comparáveis entre si. Com exceção da listagem de Belém (Novaes \& Lima 1998), nenhuma das listas acima apresenta identificação subespecífica.
Para lidar com este problema, as subespécies de aves de cada uma das listagens foram identificadas por proximidade geográfica utilizando como base o catálogo de PINTo (1978), FriEdMANN (1948) e os volumes já publicados do Handbook of Birds of the World (Del Hoyo et al. 1992, 1993, 1996, 1997, 1999, 2001, 2002, 2003, 2004). Também foram consultados inventários locais para conferir a identidade de alguns táxons (Pinto \& CAMARgo 1957, Novaes \& Lima 1991). Não foram considerados casos onde as descrições das distribuições geográficas fossem ambíguas ou tornassem difíceis a identificação dos táxons.

O uso de subespécies em análises biogeográficas é problemático, já que parte dos táxons descritos na literatura ornitológica não são unidades evolutivas e sim variações clinais subjetivas (CRACRAFT 1985). Por outro lado, um grande número de espécies que ocorrem na bacia amazônica são politípicas e parte das subespécies que as compõem são válidas. Assim, tratar espécies politípicas como entidades homogêneas tende a "uniformizar" as distribuições geográficas de entidades potencialmente importantes para análises biogeográficas e de conservação (Bates \& Demos 2001). Além disso, estudos recentes têm demonstrado que o uso de subespécies em análises biogeográficas pode ser informativo (BATEs et al. 1998).

As listagens discriminam os diferentes tipos de hábitats onde cada espécie foi registrada (campinas, matas de várzea, matas de terra firme, savanas, etc.). Nem todos os hábitats ocorrem em todas as localidades e a ausência de algumas espécies de aves num sítio pode ser explicada pela ausência de hábitat apropriado. As matas de terra firme são o único tipo de vegetação distribuído amplamente nas localidades comparadas. Nas comparações entre as listagens foram consideradas somente aves de matas de terra firme das famílias não Passeriformes e parte dos Passeriformes Suboscines (Dendrocolaptidae, Furnariidae, Thamnophilidae e Formicariidae). Estes grupos taxonômicos foram escolhidos porque existe um tratamento taxonômico mais integrado e recente para estes táxons (PINTO 1978, DEL Hoyo et al. 1992, 1993, 1996, 1997, 1999, 2001, 2002, 2003, 2004).

\section{Análises quantitativas}

Análises fenéticas e de parcimônia foram utilizadas a fim de determinar as regiões de maior similaridade com a avifauna do PNJ e estabelecer hipóteses de relações históricas entre a região oeste do Rio Negro e outras regiões da Amazônia. As listas de espécies foram comparadas através de uma análise de agrupamento com dados de presença ou ausência utilizando o índice de Jaccard como distância de similaridade e a associação pelas médias como método de ligação (McCune \& Grace 2002). A aplicação de métodos fenéticos em biogeografia baseia-se no fato de que regiões com altos níveis de similaridade podem compartilhar, ao menos parcialmente, histórias biogeográficas comuns (Rosen 1995b). Para testar a hipótese de correlação entre similaridade faunística e distância geográfica entre os sítios foi realizado um teste de Mantel. As distâncias geográficas aproximadas entre os sítios comparados foram calculadas através de um Sistema de Informações Geográficas (SIG). 
As listas de espécies foram analisadas através de uma análise de parcimônia de endemismos (PAE - do original em inglês parsimony analysis of endemicity) para identificar hipóteses de relações de área entre os sítios. As análises PAE têm sido aplicadas em estudos recentes de distribuição de vários grupos de vertebrados neotropicais como anuros (Ron 2000), lagartos (ÁvilAPires 1995), mamíferos (Fernandes et al. 1995, Silva \& Oren 1996, Costa et al. 2000) e aves (BAtes et al. 1998). Para uma revisão mais completa de estudos que aplicaram o método PAE consultar NiHEI (2006). Estes estudos se utilizaram das áreas de endemismos identificadas para aves (BATEs et al. 1998, Ron 2000), os grandes interflúvios da bacia amazônica (SILVA \& OREN 1996) ou quadrados de tamanho uniforme (Costa et al. 2000) como unidades geográficas operativas. No presente estudo foram analisadas listagens de aves que são representativas das unidades biogeográficas já identificadas para a Amazônia (Fig. 2).

A PAE tem sido aplicada em biogeografia para identificar áreas de endemismos (Morrone 1994, GÁrCiA-Barros et al. 2002) e estabelecer hipóteses de relações históricas entre áreas (SILva \& OREN 1996, BATES et al. 1998). Entre as principais críticas ao método está o fato do mesmo não levar em consideração as relações filogenéticas entre as espécies que se distribuem nas áreas analisadas e considerar a vicariância como o único processo responsável pelos padrões biogeográficos ignorando outros processos importantes como dispersão (Humphries 1989, Bisconti et al. 2001, Brooks \& van Veller 2003, Santos 2005). Apesar destas limitações, algumas características tornam a PAE um método legítimo na elaboração de hipóteses de relações entre áreas (NiHEI 2006). As relações históricas entre áreas podem ser parcialmente recuperadas através da PAE, ainda que o mesmo tenda a simplificar os cenários históricos devido a suas assunções mais restritivas (BROOKS \& VAN VeLLER 2003). Existe certa congruência entre os cladogramas de área obtidos pela PAE e aqueles obtidos através de métodos estritamente cladísticos (Cracraft \& Prum 1988, BATEs et al. 1998). Além disso, a maior parte dos processos identificados como responsáveis pelos padrões de distribuição de aves e outros vertebrados na Amazônia implica algum mecanismo de vicariância (LYNCH 1989, HAFFER 2001).

A base para as análises PAE é um matriz de espécies (caracteres) $\mathrm{x}$ regiões onde a ocorrência de uma espécie é codificada como 1 (presente) ou 0 (ausente) (Rosen 1995b, Morrone \& CRISCI 1995). Espécies que ocorrem em todos os sítios (simplesiomorfias regionais) e aquelas restritas a somente uma localidade (autapomorfias regionais) foram omitidas das análises (Rosen 1995b). Uma localidade externa sem espécies (0) foi introduzida nas análises para representar uma área ancestral hipotética (enraizamento de Lundberg cf. Rosen 1995b).

Além das análises da matriz completa de dados, foram realizadas análises individuais com aves não Passeriformes, aves Passeriformes, com as famílias Furnariidae e Dendrocolaptidae juntas e com as famílias Thamnophilidade e Formicariidae juntas. Estas análises separadas foram realizadas com o objetivo de investigar se a utilização de diferentes sub-grupos de aves resultariam em diferentes topologias nas relações históricas entre as áreas analisadas.

As análises foram feitas utilizando-se o programa PAUP* (SwOFFord 1991). As árvores de consenso foram construídas a partir da opção "50\% majority rule". A estabilidade das árvores foi acessada através do índice de decaimento (= índice ou suporte de Bremer) que pode ser interpretado como o número de passos extras necessários para "desfazer" um ramo ou relação entre táxons em um cladograma de consenso (BREMER 1994). Valores mais altos do índice de decaimento indicam relações filogenéticas e, neste caso relações entre áreas, mais estáveis (BREMER 1994). Os índices de decaimento foram calculados utilizando-se o programa Autodecay versão 5.3. Para comparar as árvores individuais foi calculado o índice de suporte total, que é a soma dos índices de decaimento de todos os ramos da árvore de consenso dividido pelo número de passos da árvore mais parcimoniosa (BREMER 1994).

\section{Biogeografia descritiva}

\section{RESULTADOS}

As distribuições geográficas de 383 táxons (espécies ou subespécies) das cerca de 470 espécies de aves registradas para a região do Parque Nacional do Jaú foram analisadas neste estudo (Anexo I). A maioria das espécies (> de 50\%) apresenta uma ampla distribuição pela Amazônia (Tab. I). Um pequeno número de aves deste grupo apresenta registros pontuais pela Amazônia, sendo algumas delas especialistas de hábitats de ocorrência restrita na bacia como savanas e campinas de areia branca (e.g. Neopipo cinnamomea (Lawrence, 1869) - Tyrannidae; Rhytipterna immunda (Sclater \& Salvin, 1873) - Tyrannidae) ou devem apresentar baixas densidades populacionais (e.g. Nyctibius aethereus (Wied, 1820) - Nyctibiidae).

A avifauna do PNJ exibe maior afinidade com aquelas encontradas nas regiões oeste e noroeste da Amazônia (Tab. I, Figs 3-6). Várias destas espécies parecem ter suas distribuições associadas à bacia do Rio Negro como Rhegmatorhina cristata (Pelzeln, 1868) - Thamnophilidae; Dolospingus fringilloides (Pelzeln, 1870) - Emberizidae e Hylophilus brunneiceps Sclater, 1866 - Vireonidae. A distribuição de algumas das espécies do noroeste da Amazônia está associada a hábitats típicos da bacia do Rio Negro como vegetações alagáveis de igapó e campinas de areia branca (e.g. Myrmeciza disjuncta Friedmann, 1945 Thamnophilidae; $H$. brunneiceps e $D$. fringilloides).

O oeste da Amazônia é outro setor com forte influência na avifauna do PNJ (Tab. I). A maioria destas espécies se distribui amplamente do oeste do Rio Negro até a alta Amazônia ao sul e norte do Rio Amazonas/Solimões nas terras baixas do Peru e Equador (e.g. Nothocrax urumutum (Spix, 1825) - Cracidae e Celeus torquatus occidentalis (Hargitt, 1889) - Picidae). Uma parte importante das espécies da alta Amazônia ocorre somente ao norte do Rio Amazonas/Solimõe (e.g. Deconychura longicauda connectens J.T. Zimmer, 1929 - Dendrocolaptidae e Dendrocolaptes certhia radiolatus Sclater \& Salvin, 1868 - Dendrocolaptidae). Um nú-

Revista Brasileira de Zoologia 24 (4): 919-940, dezembro 2007 


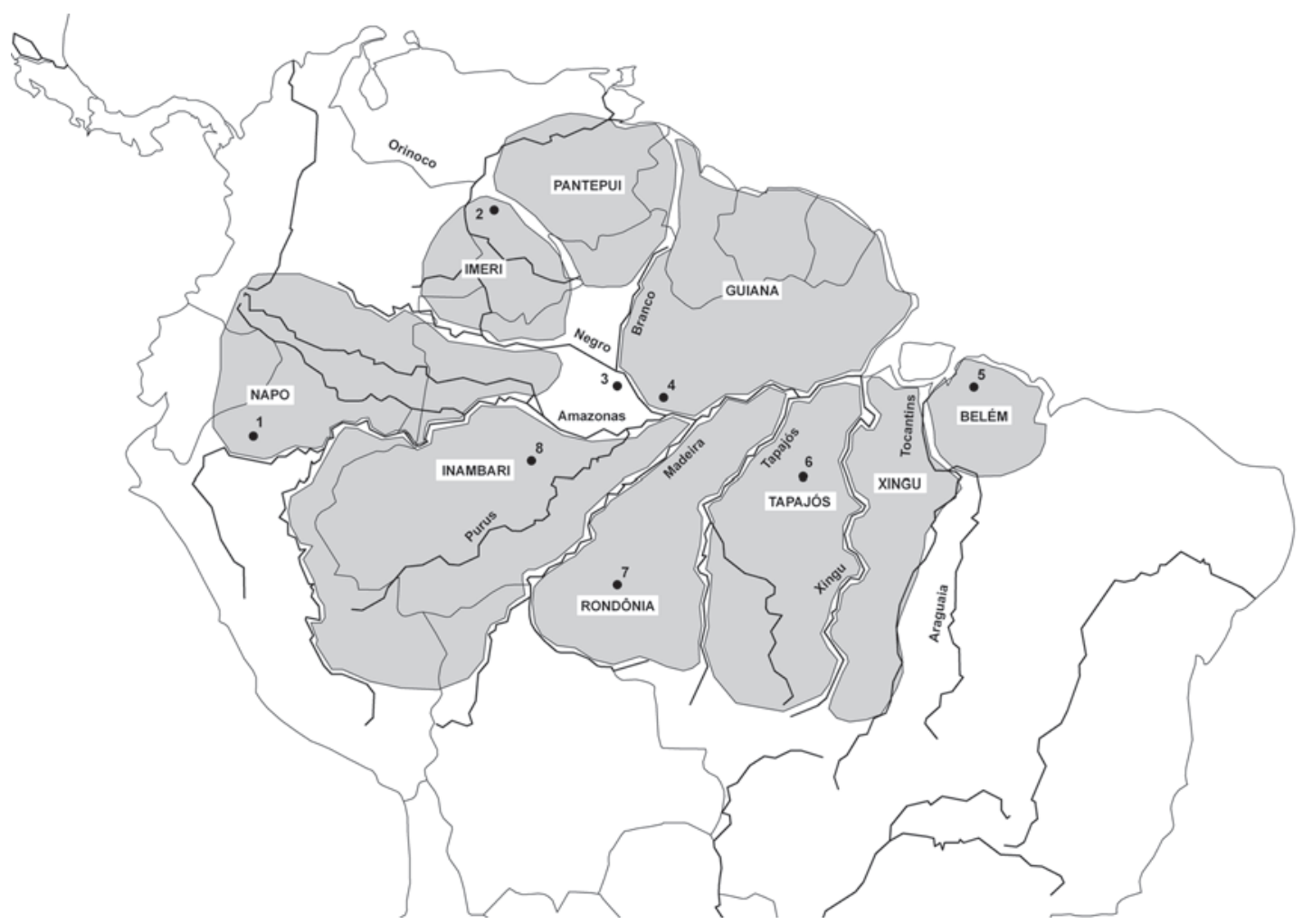

Figura 2. Área de endemismos para aves amazônicas segundo Haffer (1974), Haffer (1978), Cracraft (1985) and Silva et al. (2002) mostrando os sítios comparados neste estudo: 1) Quebrada Sucusari (Bret Whitney, com. pess.), 2) alto Rio Orinoco, Venezuela (ZıMmeR \& Hilty 1997), 3) Parque Nacional do Jaú (presente estudo), 4) Manaus (Cohn-Haft et al. 1997), 5) Belém (Novaes \& Lima 1998), 6) Alta Floresta (Zimmer et al. 1997), 7) Cachoeira Nazaré (Stotz et al. 1997) e 8) Rio Urucu (Peres \& Whittaker 1991).

Tabela I. Número de espécies de aves registradas no Parque Nacional do Jaú distribuídas nas categorias biogeográficas adotadas neste estudo.

\begin{tabular}{lr}
\hline \multicolumn{1}{c}{ Categorias de distribuição geográfica } & $\begin{array}{c}\text { Número de } \\
\text { espécies }\end{array}$ \\
\hline Espécies de distribuição ampla & $200(52 \%)$ \\
$\begin{array}{l}\text { Noroeste da Amazônia } \\
\text { Distribuição ampla ao norte do Rio }\end{array}$ & $45(11 \%)$ \\
Solimões/Amazonas & $45(11 \%)$ \\
$\begin{array}{l}\text { Noroeste e oeste da Amazônia em ambas as } \\
\text { margens do Rio Solimões/Amazonas }\end{array}$ & $38(10 \%)$ \\
$\begin{array}{l}\text { Noroeste e oeste da Amazônia ao norte do Rio } \\
\text { Solimões/Amazonas }\end{array}$ & $28(7 \%)$ \\
$\begin{array}{l}\text { Oeste da Amazônia em ambas as margens do Rio } \\
\text { Solimões/Amazonas } \\
\text { Amazônia Central entre os rios Negro e Solimões }\end{array}$ & $9(2 \%)$ \\
$\begin{array}{l}\text { Oeste da Amazônia ao norte do Rio } \\
\text { Solimões/Amazonas } \\
\text { Outros tipos de distribuição }\end{array}$ & $5(1 \%)$ \\
\hline Total de espécies & $4(1 \%)$ \\
\hline
\end{tabular}

mero restrito de espécies da alta Amazônia parece não alcançar o noroeste amazônico (e.g. Formicarius analis zamorae - Chapman, 1923 - Formicariidae e Lanio fulvus peruvianus Carriker, 1934 Thraupidae). Algumas poucas subespécies $(n=11)$ encontradas no PNJ parecem ser substituídas por outras subespécies no alto Rio Negro (Anexo I).

A avifauna do PNJ possui espécies consideradas restritas às áreas de endemismo Imeri, Napo, Inambari e Guiana (Figs 36). A área de endemismo Imeri é suportada por 18 táxons (HAFFER 1978, Cracraft 1985) sendo nove deles registrados no PNJ: Selenidera nattereri (Gould, 1836) - Ramphastidae; M. disjuncta; Myrmotherula ambigua Zimmer, 1932 - Thamnophilidae; Myrmotherula cherriei Berlepsch \& Hartert, 1902 - Thamnophilidae; $R$. cristata; Gymnopithys leucaspis lateralis Todd, 1927- Thamnophilidae; Heterocercus flavivertex Pelzeln, 1868 - Pipridae; Hylophilus $b$. brunneiceps Sclater, 1866; D. fringilloides. As áreas de endemismos do oeste da Amazônia (Napo e Inambari) também exercem uma certa influência sobre a avifauna do PNJ com treze espécies registradas na região: N. urumutum, Pionopsitta barrabandi (Kuhl, 1820) - Psittacidae; Amazona festiva festiva (Linnaeus, 1758) - Psittacidae; Topaza pyra (Gould, 1846) - 

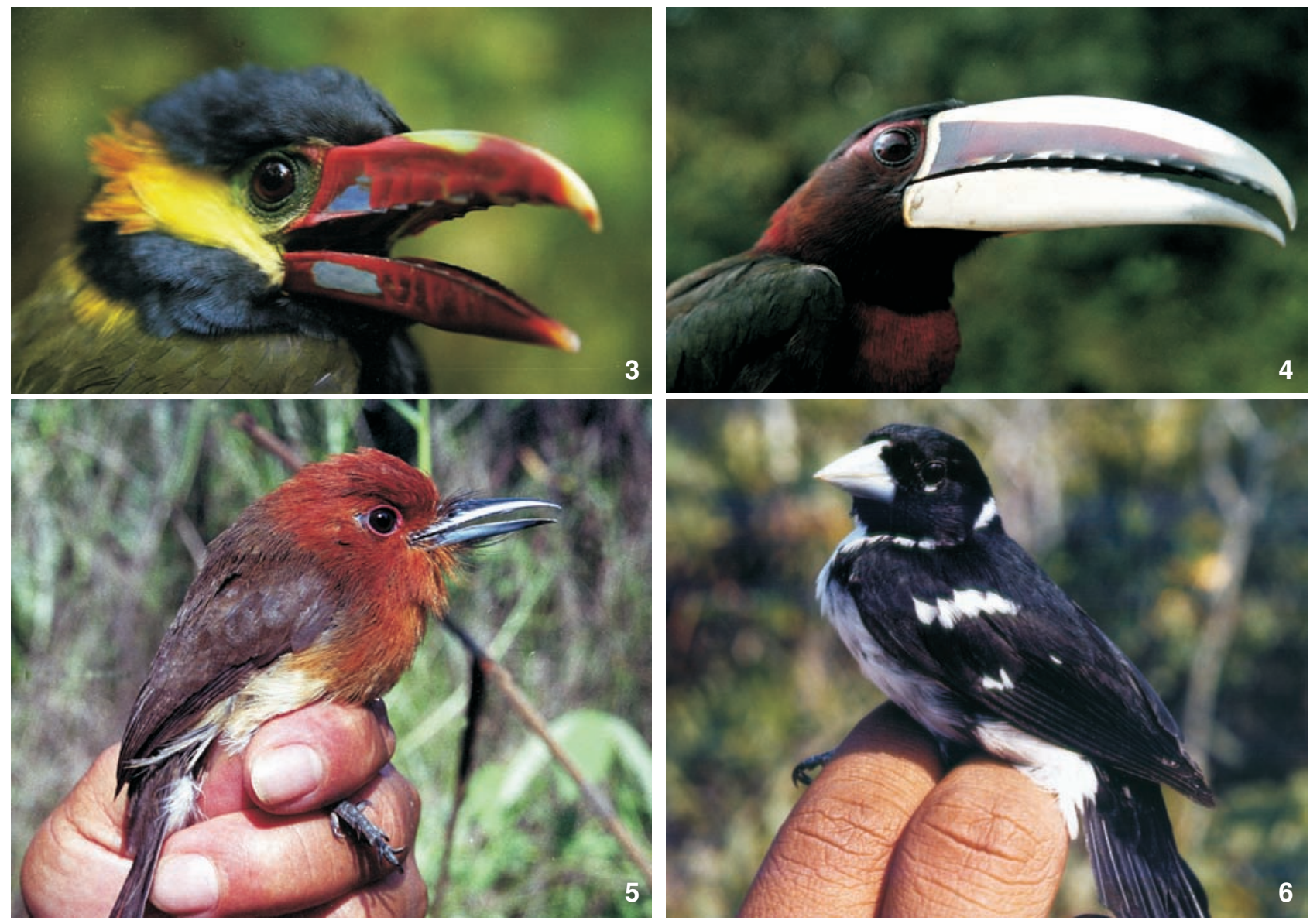

Figuras 3-6. Espécies de aves representativas das afinidades biogeográficas do Parque Nacional do Jaú: (3) Selenidera natereri, um araçari de matas de terra firme amplamente distribuído pela região noroeste da bacia amazônica. Esta é uma das espécies que dão suporte a area de endemismo Imeri, (4) Pteroglossus azara azara um táxon restrito a área de endemismo Rio Negro registrado em vários hábitats como matas de terra firme, campinaranas e matas de igapó, (5) Nonnula amaurocephala um joão-bobo especializado em matas de igapó do baixo Rio Negro, (6) Dolospingus fringilloides um papa-capim especializado em campinas e igapós abertos ao longo do Rio Negro.

Trochilidae; Pharomachrus pavoninus (Spix, 1824) - Trogonidae; Eubucco richardsoni (Gray, 1846) - Capitonidae; Dendrexetastes rufigula devillei (Lafresnaye, 1850) - Dendrocolaptidae; Frederickena unduligera (Pelzeln, 1868) - Thamnophilidae; Myrmotherula assimilis Pelzeln, 1868 - Thamnophilidae; Hypocnemis hypoxantha Sclater, 1869 - Thamnophilidae; Attila citriniventris Sclater, 1859 - Tyrannidae; Phoenicircus nigricollis Swainson, 1832 - Cotingidae; Ramphocelus nigrogularis Spix, 1825 - Thraupidae. Poucos táxons de distribuição considerada restrita à região biogeográfica da Guiana foram registrados no PNJ (e.g. Polioptila guianensis Todd, 1920 - Sylviidae; Pachyramphus surinamus (Linnaeus, 1766) - Tyrannidae).

\section{Biogeografia quantitativa}

Três unidades biogeográficas foram identificadas na análise de agrupamento (Fig. 7). As avifaunas dos sítios localizados ao

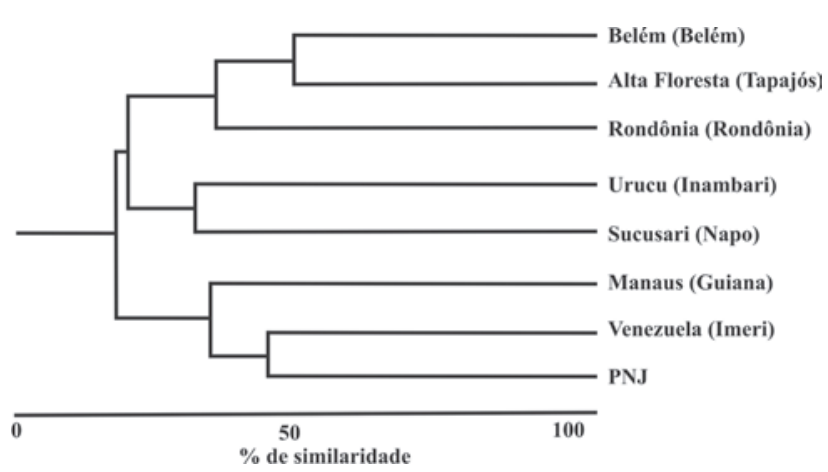

Figura 7. Padrão de agrupamento de oito localidades na Amazônia baseado na similaridade de composição de espécies de aves usando dados de presença ou ausência. Entre parênteses estão as áreas de endemismo mostrado na figura 2.

Revista Brasileira de Zoologia 24 (4): 919-940, dezembro 2007 
sul do Rio Amazonas (Belém, Alta Floresta e Rondônia) apresentam uma similaridade relativamente alta; especialmente Belém e Alta Floresta. Os sítios da alta Amazônia (Quebrada Sucusari e alto Rio Urucu) formam o segundo grupo e os sítios localizados ao norte do Rio Amazonas (com exceção de Quebrada Sucusari) se agrupam na terceira unidade biogeográfica (Fig. 7).

A avifauna do PNJ apresenta uma alta similaridade com o noroeste da Amazônia (sítio do alto Rio Orinoco), um resultado similar ao encontrado nas análises descritivas. As avifaunas do PNJ e do alto Rio Orinoco compartilham 45\% de suas espécies sendo as regiões com maior similaridade faunística (Fig. 8). A proporção de espécies compartilhadas entre o PNJ e outras regiões da Amazônia varia de 17\% a 30\% (Fig. 8).

Existe uma correlação significativa entre as distância geográficas e as similaridades faunísticas (calculada pelo índice de Jaccard) nos sítios analisados (teste de Mantel, $r=-0,38, p=0,02$ ). Os sítios com menor similaridade foram os mais distantes entre si (Quebrada Sucusari e Belém). Por outro lado, sítios relativamente próximos (< de $500 \mathrm{~km}$ ) como PNJ e Urucu tiveram níveis mais baixos de similaridade do que sítios mais distantes (> de $1000 \mathrm{~km}$ ) como Alta Floresta e Belém. Este resultado sugere que, ainda que a distância entre sítios explique parte (38\%) da similaridade faunística, é possível que a composição das espécies esteja sendo influenciada por fatores históricos já que os sítios comparados estão em áreas de endemismos distintas (Fig. 2).

\section{Cladogramas de área}

As análises de parcimônia de endemismo (PAE) revelaram padrões complexos e nem sempre consistentes entre si. $\mathrm{Na}$ análise total dos dados com 281 espécies (caracteres) foram identificados três grupos bem definidos (Fig. 9). Os valores dos índices de decaimento foram muito baixos, indicando uma baixa estabilidade nas relações entre as áreas.

Analisando somente as aves não Passeriformes, dois grupos bem distintos foram identificados: um localizado ao sul do Rio Amazonas (com exceção de Quebrada Sucusari) e outro grupo localizado ao norte do Rio Amazonas (Fig. 10). Novamente, o PNJ se posiciona como grupo-irmão de Manaus e Venezuela (Fig. 10). Neste caso, os índices de decaimento são relativamente altos nos clados Alta Floresta e Belém e nos clados que reúnem os sítios ao norte do Rio Amazonas.

Os padrões de relação entre áreas são totalmente diferentes, considerando somente aves Passeriformes com três grupos bem definidos (Fig. 11). Neste caso, o PNJ se agrupa com os sítios localizados no oeste da Amazônia (Quebrada Sucusari) tendo o sítio Urucu posicionado como grupo-irmão (Fig. 11). Os baixos índices de decaimento, entretanto, indicam uma acentuada instabilidade nas relações entre as áreas.

Nas análises da distribuição das famílias Furnariidae e Dendrocolaptidae, os sítios PNJ, Sucusari e Urucu formam uma tricotomia e os baixos índices de decaimento indicam um suporte muito fraco para os clados encontrados (Fig. 12). O cladograma de área que recebeu maior suporte envolve as famílias Thamnophilidae e Formicariidae (Fig. 13). Neste caso, três grupos bem distintos foram detectados e o PNJ se agrupa com a Venezuela, sendo Manaus o grupo-irmão deste clado (Fig. 13). Esta árvore apresentou uma topologia muito similar a análise de agrupamento, com exceção das posições do sítios Rondônia, Belém e Alta Floresta (comparar Figs 7 e 13).

Uma análise comparativa mostra que os cladogramas de área obtidos com de aves não Passeriformes (Fig. 10) e Thamnophilidae e Formicariidae juntos (Fig. 13) receberam os maiores suportes e que a árvore com todos os dados (Fig. 9) foi a que apresentou menor estabilidade (Tab. II). Além disso, o cladograma construído com dados de Thamnophilidae e Formicariidae recebeu os maiores índices de consistência e de retenção (Fig. 13).

\section{Aves endêmicas do centro-oeste da Amazônia}

Durante as análises de distribuição das aves foram identificados seis táxons aparentemente endêmicos do setor oeste do Rio Negro, não alcançando o noroeste nem os setores da alta Amazônia. Alguns destes táxons são bem diferenciados apresentando caracteres diagnosticáveis, enquanto outros precisam de análises comparativas mais detalhadas para definir seus "status" taxonômicos.

\section{DISCUSSÃO}

\section{Afinidades biogeográficas na avifauna do PN]}

O PNJ está localizado em uma região intermediária entre as grandes unidades biogeográficas da Amazônia e sua avifauna mostra uma acentuada afinidade com os setores noroeste e oeste da bacia amazônica (Tab. I, Figs 3-6 e 8). Desde modo, o centro-oeste da Amazônia pode ser caracterizada como uma região composta do ponto de vista da biogeografia já que espécies de diferentes setores da bacia são encontradas juntas, além de contar com espécies de distribuições restritas.

Table II. Medidas de estabilidade nos cladogramas de área obtidos como diferentes bases de dados

\begin{tabular}{lcccc}
\hline \multicolumn{1}{c}{ Base de dados } & Número de táxons & Número de passos (S) & Suporte total (T) & Índice de suporte total (T/S) \\
\hline Todas as espécies & 281 & 539 & 8 & 0,015 \\
Somente não Passeriformes & 181 & 354 & 29 & 0,082 \\
Somente Passeriformes & 101 & 175 & 9 & 0,051 \\
Furnariidae mais Dendrocolaptidae & 48 & 85 & 5 & 0,059 \\
Thamnophilidae mais Formicariidae & 50 & 83 & 17 & 0,205 \\
\hline
\end{tabular}

Revista Brasileira de Zoologia 24 (4): 919-940, dezembro 2007 


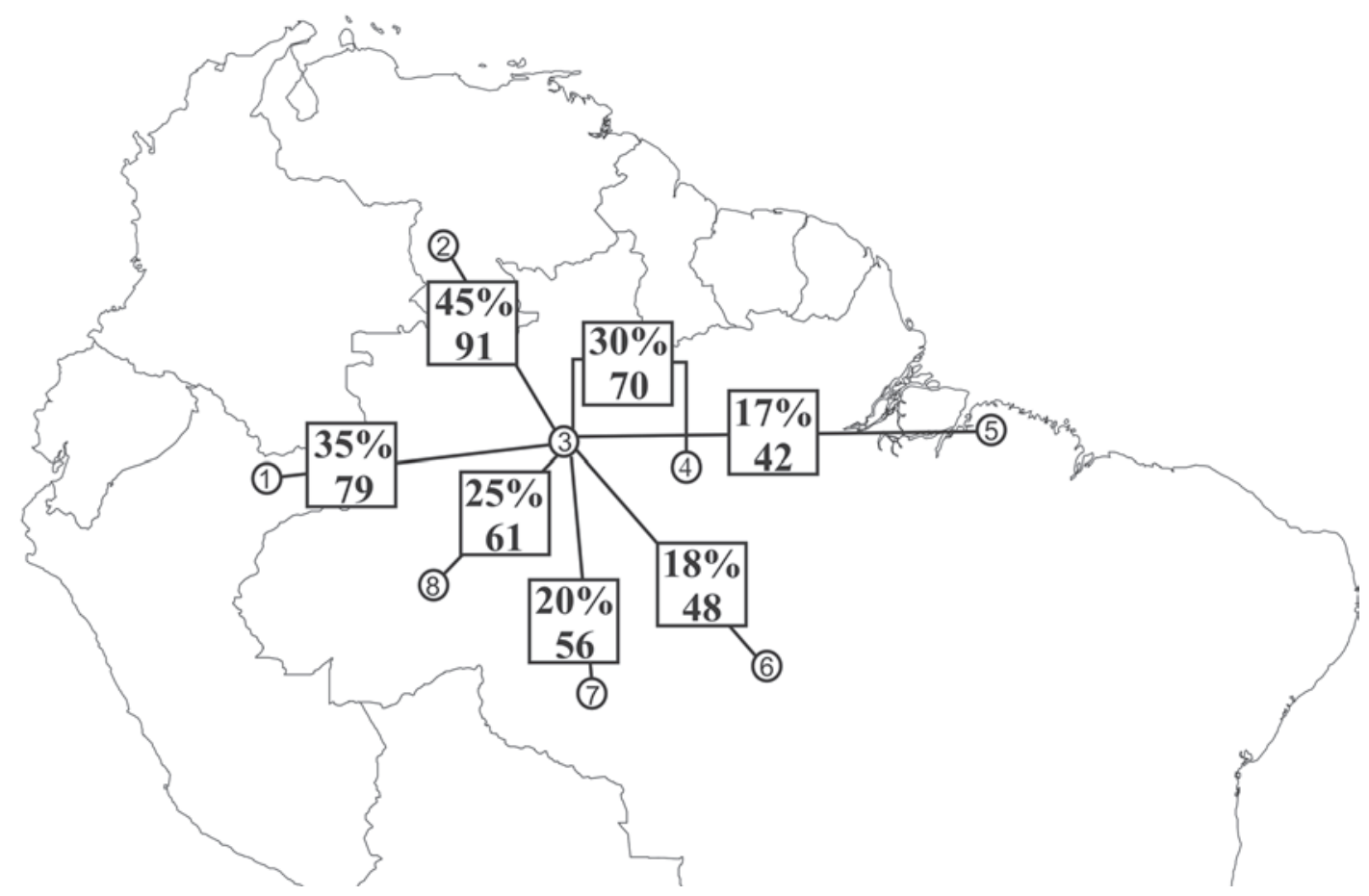

Figura 8. Similaridade avifaunística entre o Parque Nacional do Jaú e outras localidades da bacia amazônica. As localidades estão numeradas como na figura 2. O número na parte de cima da caixa é a percentagem de similaridade calculada através do índice de Jaccard e na parte de baixo estão os número de espécies compartilhadas entre as localidades ligadas.

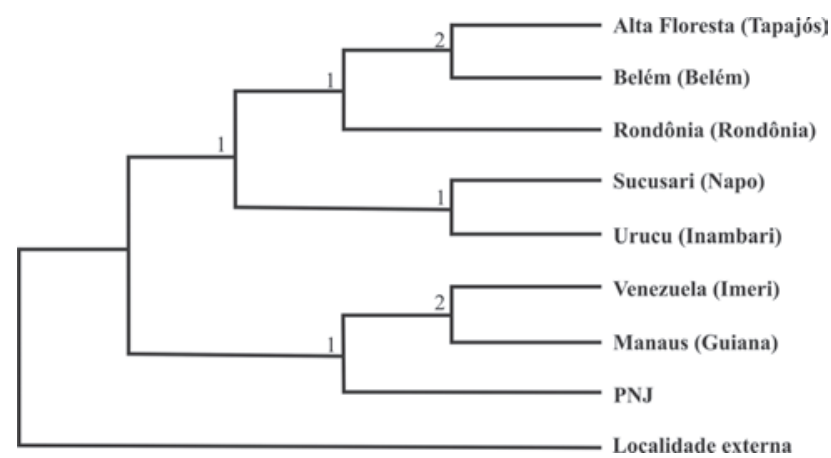

Figura 9. Cladograma de area resultado da análise de parcimônia de endemismo (PAE) incluíndo aves não Passeriformes e Passeriformes das famílias Furnariidae, Dendrocolaptidae, Thamnophilidae e Formicariidae. Os números acima de cada ramo são os índices de decaimento. Índice de consistência $=0,52$; índice de retenção $=0,45$.

A maioria das espécies de aves de interesse biogeográfico registradas no PNJ se distribui amplamente no setor delimitado pelos rios Negro e Solimões, os rios mais importantes para a definição das distribuições geográficas das aves amazônicas (Haffer 1992). Aproximadamente 50-70 pares de espécies de aves aparentadas se substituem em margens opostas do Rio
Negro em seu baixo curso (Haffer 1992). Além disso, o Negro é um dos rios mais importantes para a distribuição geográfica de primatas na Amazônia devido a características hidrológicas como distância entre margens e estabilidade do curso (Ayres \& Clutton-Brock 1992). Um menor número de espécies de aves é compartilhado entre o PNJ e Manaus, sítios separados por cerca de $200 \mathrm{~km}$ em margens opostas do Rio Negro, do que entre o PNJ e o alto Rio Orinoco na Venezuela distantes mais de 1000 $\mathrm{km}$ em linha reta (Fig. 8). Neste caso, a similaridade na composição de espécies não esta relacionada à proximidade geográfica, mas ao grande poder de isolamento do Rio Negro em seu baixo curso.

A capacidade de um rio separar populações animais varia ao longo de seu canal. Populações isoladas na foz dos rios potencialmente podem entrar em contato nas regiões de cabeceiras (HAFFER 1992). Intercâmbio de espécies de aves entre margens opostas é provável de ocorrer nas cabeceiras do Rio Negro com acontece no alto curso dos rios Madeira e Tapajós (HAFFer 1992). A mistura de avifaunas no alto Rio Negro é ilustrada pelos registros de $S$. natereri (espécie do oeste do Rio Negro) e Gymnopithys rufigula (Boddaert, 1783) - Thamnophilidae (espécie do leste do Rio Negro) em um mesmo sítio no alto Rio Orinoco (Zimmer \& Hitтy 1997). Outros exemplos de pares de espécies separadas no baixo curso do Rio Negro que se encontram e, em alguns casos se hibridizam, no alto curso incluem Pionopsitta barrabandi (Kuhl, 


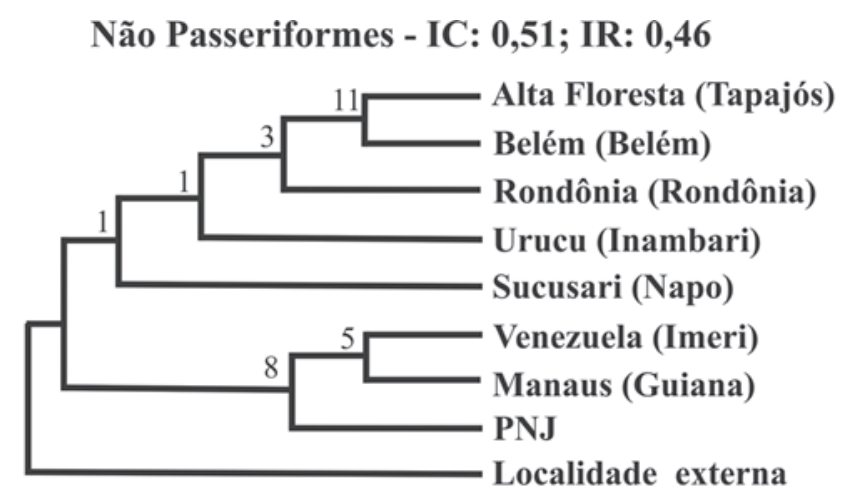

Furnariidae + Dendrocolaptidae

- IC: 0,58; IR: 0,57

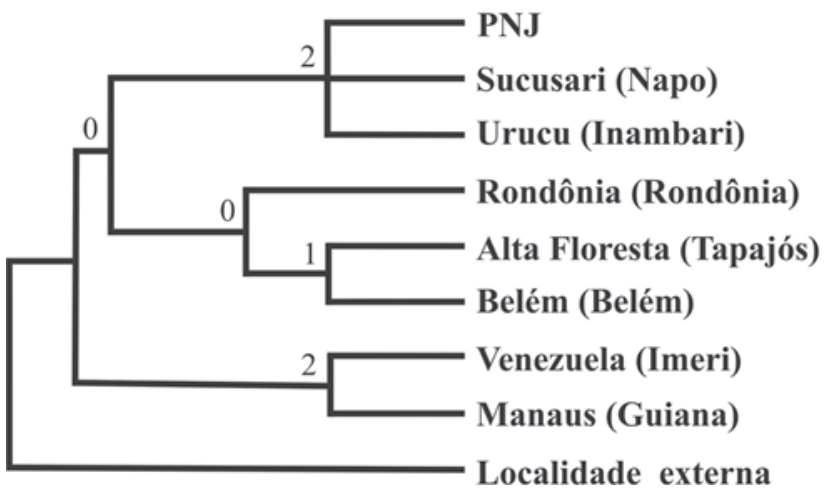

Passeriformes - IC: 0,58; IR: 0,50

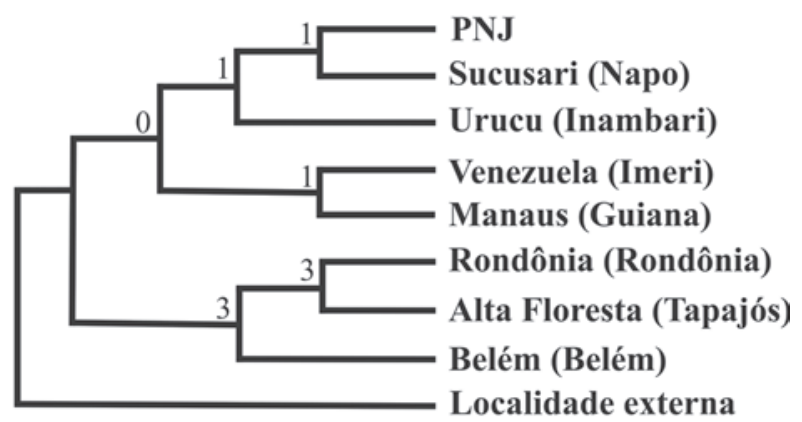

Thamnophilidae + Formicariidae

- IC: 0,61; IR: 0,53

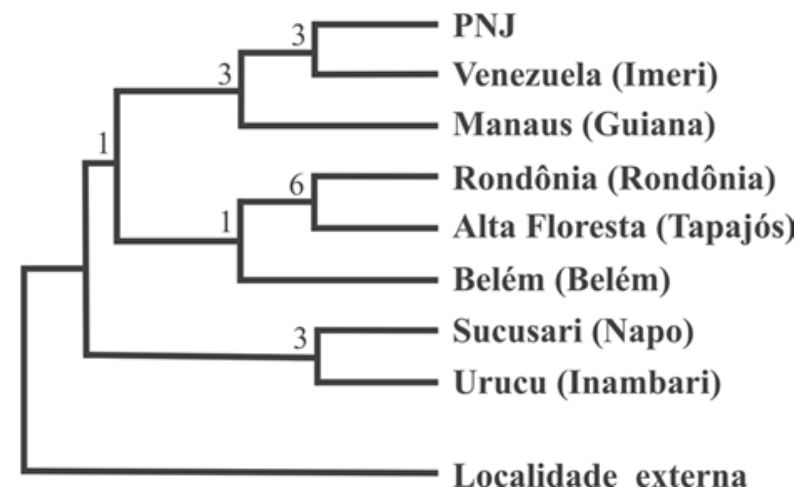

Figuras 10-13. Cladogramas de área resultado da análise de parcimônia de endemismo (PAE) usando diferentes sub-grupos de espécies: (10) espécies não Passeriforme, (11) espécies Passeriformes das famílias Furnariidae, Dendrocolaptidae, Thamnophilidae e Formicariidae, (12) Furnariidae mais Dendrocolaptidae, (13) Thamnophilidae mais Formicariidae. Os números acima de cada ramos são os índices de decaimento. (IC) índice de consistência, (IR) índice de retenção.

1820)/Pionopsitta caica (Latham, 1790) - Psittacidae e Ramphastos tucanus tucanus Linnaeus, 1758/Ramphastos tucanus cuvieri Wagler, 1827 - Ramphastidae (HAFFer 1992).

O Amazonas é outro rio importante para as características biogeográficas da avifauna do PNJ. As distribuições de cerca de 150 espécies de aves coincidem com o Amazonas na altura da foz do Rio Negro (Haffer 1992). Este número de espécies diminui gradativamente em direção às cabeceiras (HAFFER 1992). O grupo biogeográfico formado por Quebrado Sucusari (norte do Rio Amazonas) e o alto Rio Urucu (sul do Amazonas) (Fig. 8) provavelmente é resultado desta diminuição no poder de isolamento do Amazonas nas cabeceiras.

\section{Biogeografia do interflúvio Negro/Solimões}

Apesar de um grande número de espécies de aves se distribuir de modo contínuo pelo oeste do Rio Negro, esta região não parece ser tão uniforme do ponto de vista biogeográfico. Algumas espécies de aves ocorrem no noroeste da Amazônia (alto
Rio Negro, Rio Orinoco etc.), mas aparentemente não alcançam a porção oeste da bacia (e.g. Dendrocincla fuliginosa phaeochroa Berlepsch \& Hartert, 1902 - Dendrocolaptidae; Hylophylax poecilinotus duidae Chapman, 1923 - Thamnophilidae), enquanto outras espécies registradas no oeste amazônico não alcançam o região noroeste (e.g. Odonthophorus gujanensis buckley Chubb, 1919 - Phasianidae, Dendrocincla fuliginosa neglecta Todd, 1948 Dendrocolaptidae).

A avifauna que ocorre nas campinas do PNJ também parece exibir um padrão biogeográfico similar ao das aves florestais. Estudos recentes realizados em vegetações sobre solos arenosos no Peru, incluindo vegetações similares às campinas do Rio Negro, revelaram a ocorrência de várias espécies novas de aves (Whitney \& Alonso 1998, Alonso \& Whitney 2001, Isler et al. 2001). Infelizmente, poucos estudos ornitológicos foram feitos neste tipo de ambiente (Alonso \& Whitney 2003, Borges 2004, Poleto \& Aleixo 2005) dificultando o estabelecimento mais detalhado das distribuições geográficas destas espécies. 
Entretanto, é possível hipotetizar que estes táxons recém-descritos sejam endêmicos das vegetações sobre solos arenosos do oeste da Amazônia não ocorrendo nas campinas e campinaranas do Rio Negro. De fato, tentativas de encontrar estas espécies nas campinas e campinaranas do PNJ não foram bem sucedidas. É possível que as campinas e campinaranas do oeste e do noroeste da Amazônia tenham se separado tempo suficiente para estes táxons terem se divergido em populações distintas.

A distribuição geográfica das espécies de aves florestais e não florestais sugere que exista uma discontinuidade biogeográfica em algum lugar entre os setores oeste e o noroeste da Amazônia. Quais características físicas do ambiente poderiam auxiliar na delimitação desta região de substituição geográfica entre espécies e subespécies de aves nesta região? Como mencionado acima, os rios são importantes para identificar os limites de distribuição geográfica das espécies de aves na Amazônia. Ao menos dois grandes rios localizados no oeste amazônico ao norte do Amazonas poderiam afetar a distribuição das aves: o Japurá e o Iça. Estes dois rios correm paralelos ao Rio Negro em direção a noroeste (Fig. 1). Foi sugerido que o Rio Putumayo (nome peruano do Rio Iça) coincida com o limite de substituição geográfica de Percnostola rufifrons jensoni Capparella et al., 1997 - Thamnophilidae e Percnostola rufifrons minor Pelzeln, 1868 - Thamnophilidae (CAPARELla et al. 1997). Infelizmente, a avifauna das regiões próximas destes dois rios é pobremente amostrada (Oren \& Albuquerque 1991, Oren 2001). Existe, ainda, a possibilidade de que a região de contato secundário entre as espécies e subespécies de aves ocorra em regiões interfluviais de matas de terra firme sem estar relacionada ao sistema hidrográfico, como acontece no sul do Rio Amazonas (HAFFer 1992). Neste caso, diferenças sutís nos tipos de matas onde estas espécies ocorrem podem ser percebidas como barreiras pelas aves.

\section{As áreas de endemismo na Amazônia e a avifauna do PN]}

A delimitação de áreas ou centros de endemismo é uma das mais importantes etapas de uma análise biogeográfica já que permite reconhecer as unidades básicas da estrutura histórica na qual as biotas evoluíram (CRACRAFT 1988). Nas terras baixas da Amazônia foram identificadas oito áreas de endemismo suportadas pela distribuição restrita de um variado número de espécies de aves (Haffer 1974, 1978, Cracraft 1985, Silva et al. 2002). A maioria destas áreas de endemismos se distribui em regiões periféricas da bacia amazônica e tem seus limites definidos pelos grandes rios da bacia (Fig. 2).

O PNJ ocupa uma região central entre as áreas de endemismo da Amazônia e sofre uma acentuada influência da área de endemismo Imeri. Como descrito originalmente por HAFfer $(1969,1974,1978)$, os limites geográficos desta área coincidem com o noroeste do Brasil, sul da Venezuela e sudeste da Colômbia. Aproximadamente 18 taxóns dão suporte a área de endemismo Imeri (HAFFer 1978, Cracraft 1985) sendo que vários deles foram registrados mais ao sul no PNJ, indicando que os limites geográficos do Imeri se extendam até o norte do Rio Amazonas como proposto por Cracraft (1985).
Uma boa parte das espécies de aves que dão suporte a área de endemismo Imeri são depedentes, em variados níveis, de campinas e matas de igapó, hábitats típicos da bacia do Rio Negro (Anderson 1981, Pires \& Prance 1985, Borges 2004). As espécies $M$. disjuncta, Myrmotherula cherriei, $H$. flavivertex, $H$. brunneiceps e $D$. fringilloides foram registradas em campinas de areia branca e em matas de igapó mais abertas no PNJ e em outros sítios da bacia do Rio Negro (Prum et al. 1996, Zimmer \& Hilty 1997, Borges \& Carvalhaes 2000, Borges \& Almeida 2001, Borges 2004, NAKA et al. 2006). Outras espécies consideradas restritas a área Imeri como Thripophaga cherriei Berlepsch \& Hartert, 1902 - Furnariidae e Percnostola caurensis (Hellmayr, 1906) - Thamnophilidae, apesar de pouco conhecidas na natureza, parecem ser especialistas de matas alagadas e matas que se desenvolvem sobre afloramentos rochosos (Ridgely \& Tudor 1994, Zimmer 1999, Hilty 2003). A distribuição de boa parte das espécies de aves que dão suporte a área de endemismo Imeri está condicionada à ocorrência de hábitats e micro-habitats específicos, o que reforça a importância de fatores ecológicos atuais para explicar a distribuição geográfica das espécies na Amazônia (Tuomisto \& Ruokolainen 1997).

Neste estudo também foi identificada uma nova área de endemismo na região centro-oeste da Amazônia. HAFFer \& FITZPATRICK (1985) já haviam chamado a atenção para a distribuição de um pequeno número de espécies de aves que parecem restritas à Amazônia Central. Amorin \& Pires (1999) também identificaram uma pequena área de endemismo a oeste do Rio Negro, mas sem oferecer detalhes sobre quais espécies dão suporte a este componente biogeográfico (АMORIM \& PirEs 1999: fig. 27).

\section{Relações entre as áreas biogeográficas da Amazônia}

Padrões de relação biogeográfica entre áreas nos diferentes estudos com vertebrados amazônicos revelaram consistência em algumas associações. As áreas de endemismos Napo e Inambari parecem formar um clado consistente em todos os estudos realizados com diferentes conjuntos de dados, grupos taxonômicos e abordagens metodológicas (Cracraft \& PrUm 1988, Bates et al. 1998, Silva \& Oren 1996, Ron 2000, este estudo). Em contraste, a posição relativa das áreas ao sul do Rio Solimões/Amazonas (Rondônia, Tapajós e Belém) exibem diferentes topologias nos cladogramas de área. Em algumas análises deste estudo, Rondônia se posiciona como grupo-irmão de Belém e Tapajós (Fig. 10) e em outros casos é Belém que se posiciona como grupo-irmão de Tapajós e Rondônia (Fig. 11). Estes resultados são consistentes com os obtidos por BATEs et al. (1998).

Uma das grandes diferenças nos resultados obtidos em diferentes estudos é a posição da área de endemismo Imeri. A distribuição de espécies e subespécies de aves Passeriformes mostra que Imeri se posiciona como grupo-irmão do clado (Napo(Inambari) na maioria dos cladogramas obtidos (BATES et al. 1998). Resultados similares foram obtidos por Cracraft \& Prum (1988), analisando diferentes táxons de papagaios 
(Psittacidae) e tucanos (Ramphastidae) numa abordagem filogenética. A distribuição de primatas revela que Imeri forma um clado não resolvido (tricotomia) com Inambari e Napo (SILVA \& Oren 1996). Na maioria dos cladogramas obtidas no presente estudo, a área de endemismo Imeri forma um clado com a área Guiana (Figs 9-12). A única exceção é a árvore obtida com dados de distribuição de Thamnophilidade e Formicariidae, onde o Imeri forma uma clado com o PNJ (área de endemismo Rio Negro) com Guiana se posicionando como grupo-irmão deste (Fig. 13). Vale lembrar que esta árvore recebeu o maior índice de suporte total (Tab. II).

Porque existem incongruências entre os diferentes estudos e grupos taxonômicos analisados? Este estudo e o de BATES et al. (1998) apresentam algumas similaridades analíticas e uma comparação entre eles pode ser informativo. Apesar de ambos os estudos se utilizaram do PAE com método de análise, existem algumas importantes diferenças entre os mesmos. Nas análises de Bates et al. (1998) foram utilizados todos os táxons, mesmo aqueles não informativos que ocorreram em todas as áreas de endemismo ou somente em uma delas. Esta opção aumentou artificialmente o número de caracteres (taxóns) utilizados nas análises. BATEs et al. (1998) também não levaram em consideração as diferenças no uso de hábitats pelas espécies e a codificação de ausência de uma espécie poderia ser atribuída a ausência de hábitats apropriados. É possível que estas diferenças na abordagem analítica tenham afetado as relações entre as áreas analisadas.

BAtes et al. (1998) também chamaram a atenção para o fato de que a própria inconsistência das bases de dados pode afetar, de modo não esclarecido, as conclusões dos estudos. Codificar uma espécie como ausente de um sítio pode ser problemático já que o conhecimento sobre a distribuição de aves na Amazônia ainda é incompleto, apesar dos recentes avanços (Oren \& Albuquerque 1991, Oren 2001). Além disso, hipóteses de relações filogenéticas não estão disponíveis para a maioria das espécies de aves amazônicas e o arranjo taxonômico ainda é baseada em estudos sistemáticos antigos. Assim, as bases de dados estão "contaminadas" por táxons que não são entidades consistentes, mas meras variações clinais. Estes problemas só serão resolvidos através da ampliação das coletas de aves na região e dos estudos taxonômicos e biogeográficos de espécies individuais.

Outras possíveis explicações para estas incongruências podem não estar relacionadas a limitações metodológicas ou análiticas e envolver cenários biogeográficos mais complexos. Múltiplas conexões e separações entre áreas distintas em fases diversas do tempo geológico podem ter resultado em uma mistura de eventos históricos (Cracraft 1988, 1994, Bates et al. 1998). As incongruências nos cladogramas de áreas obtidos nos diferentes grupos ou sub-grupos de espécies encontradas no presente estudo (e.g. Figs 10, 11 e 13) e em outros, sugerem uma complexa história de diversificação da avifauna Amazônica (Bush 1994, Bates et al. 1998).

\section{Possíveis mecanismos de vicariância influenciando a dis- tribuição de aves no noroeste da Amazônia}

No noroeste e oeste da bacia amazônica são encontradas três áreas de endemismos para aves: Imeri no noroeste extremo, Napo mais a oeste e Rio Negro na região central (Fig. 2). Quais processos de vicariância podem ter levado à diferenciação das avifaunas encontradas nestas unidades biogeográficas? A história geológica das regiões noroeste e oeste da Amazônia, incluindo sítios amostrados na Colômbia, Peru e Brasil tem sido amplamente estudada (HoORN 1993, 1994a,b, RASäNEN et al. 1995, LUNDBERg et al. 1998) permitindo a elaboração de alguns cenários biogeográficos hipotéticos.

Os eventos geológicos melhor estudados nesta região dizem respeito à penetração do oceano Pacífico e caribenho no interior da alta Amazônia, o soerguimento da cordilheira dos Andes e o estabelecimento do atual sistema de drenagem da bacia amazônica. Todos estes eventos ocorreram ao longo do Mioceno, ou seja, entre 5 e 25 milhões de anos antes do presente (AP). Hoorn (1993) resume a série de eventos geológicos que ocorreram no oeste e noroeste da Amazônia nas etapas descrita a seguir. No ínicio do Mioceno, o sistema fluvial drenava no sentido leste-oeste transportando sedimentos originários do escudo das Guianas. Neste período, incursões marinhas esporádicas podem ter alcançado a região através do proto-Orinoco e de um portal provavelmente localizado na região oeste dos Andes. Durante o Mioceno médio a direção de deposição dos sedimentos foi alterada para o sentido oeste-leste devido ao soerquimento do setor leste da cordilheira andina, estabelecendo o atual sentido da drenagem na bacia. Os sedimentos depositados no noroeste da Amazônia tinham como fonte o leste da Cordilheira dos Andes. Neste período foi estabelecido um sistema fluvio-lacustre com influência estuarina devido à continuidade das incursões marinhas através da conexão com o mar do Caribe. Do final do Mioceno ao início do Plioceno a conexão entre a alta Amazônia e o mar do Caribe deve ter sido fechada devido ao intenso soerguimento dos Andes dando origem ao atual sistema de deposição de sedimentos e da malha hidrográfica. Este período também coincidiu com uma queda do nível do mar em escala global (MORNer et al. 2001).

Grandes quantidades de sedimentos andinos foram depositadas no noroeste e oeste da Amazônia do médio ao final do Mioceno e o paleo-ambiente regional foi extensivamente dominado por rios, lagos e brejos com predomínio de palmeiras (e.g. Mauritia - Arecaceae), plantas associadas a ambientes ribeirinhos (e.g. Amanoa e Alchornea - Euphorbiaceae), capins e outras herbáceas flutuantes (Hoorn 1994a). Esta paisagem alagadiça teve influência marinha intermitente documentada pela presença de pólen fossilizado de Rhizophora (Rhizophoraceae), foraminíferos, moluscos e peixes de origem marinha nos sedimentos analisados (Nuttal 1990, Hoorn 1994b, Lundberg et al. 1998). NutTal (1990) descreve a região da alta Amazônia durante o Mioceno como sendo uma região de mais de $500 \mathrm{~km}$ de extensão ocupada por pântanos, lagos e rios com diferentes níveis de salinidade

Revista Brasileira de Zoologia 24 (4): 919-940, dezembro 2007 
devido a conexões intermitentes com o mar do Caribe. LunDBERG et al. (1998) interpretam a paleo-paisagem desta mesma região como sendo formada por um extensivo sistema de água doce, com variados graus de salinidade, composto de lagos permanentes denominados em seu conjunto de "Lago Pebas".

Como a história de diversificação da avifauna das regiões oeste e noroeste da Amazônia foi influenciada pelos eventos geológicos descritos acima? Os extensos alagados registrados no Mioceno da alta Amazônia, incluindo o "Lago Pebas", podem ter isolado o setor noroeste da Amazônia (área Imeri) das regiões que ocupavam terrenos em cotas mais altas do oeste da Amazônia (áreas Napo e Inambari) que não tenham sido inundadas durante as transgressões marinhas que se misturaram aos acúmulos de água doce vinda dos Andes. As incursões marinhas que alimentavam as áreas alagadas da alta Amazônia terminaram no final do Mioceno (Hoorn 1993, Lundberg et al. 1998). Assim a avifauna das áreas de endemismo Napo e Inambari pode ter se separado da avifauna da área de endemismo Imeri à cerca de 8-10 milhões de anos.

Um aspecto importante deste potencial mecanismo de vicariância é a extensão de área que deve ter sido alagada pelas incursões marinhas em associação com o sistema de água doce. Devido ao baixo relevo, boa parte das regiões noroeste e oeste da Amazôzia deve ter sido submersa inclusive as áreas Napo e Inambari (BATEs 2001). É possível, entretanto, que porções de terreno não alagados tenham se distribuído nas regiões mais próximas da recém-formada cordilheira do Andes onde um relevo um pouco mais elevado tenha possibilitado a ocorrência de vegetação de terra firme onde as aves podem ter se refugiado. Bates (2001) hipotetizou, também, que durante as incursões marinhas do Mioceno, a área Imeri estaria submersa, mas o arco de Vaupés localizado no noroeste da Amazônia, pode indicar uma região com porções mais altas de relevo (LUNDBERG et al. 1998).

Análises filogenéticas moleculares de mutuns e jacutingas (Cracidae) e curicas (gênero Pionopsitta - Psittacidae) sugerem que incursões marinhas, eventos tectônicos e de dinâmica de rios que ocorreram durante o Mioceno médio tiveram um importante papel na história de diversificação destes grupos (PEReira \& BaKer 2004, Grau et al. 2005, Ribas et al. 2005). Incursões marinhas também têm sido indicadas como os principais eventos de vicariância associados à especiação de raias (família Potamotrygonidae) da América do Sul (Lovejoy et al. 1998).

Se este mecanismo estiver correto, a especiação das aves do noroeste/oeste da Amazônia deve ter ocorrido em um período de tempo muito mais antigo do que o preconizado por alguns biogeógrafos que acreditam que o Pleistoceno tenha sido o período em que a maioria das espécies tenha surgido na Amazônia (Haffer 1969). De fato, estudos realizados com aloenzimas e DNA mitocondrial de algumas poucas espécies de aves e mamíferos amazônicos sugerem períodos mais antigos do que o Pleistoceno para a divesificação destes grupos de espécies (Moritz et al. 2000). Datações de eventos de especiação em aves do gênero Gymnopithys Bonaparte, 1857 - Thamnophilidae e Ramphocelus Desmarest, 1805 - Thraupidae (НАСКETT 1993, 1996) e mães-da-lua da família Nyctibidae (BRUMFIeld et al. 1997) variam de 2.35 a 7 milhões de anos AP, o que coincide, grosso modo, como o final do Mioceno e início do Plioceno.

O estabelecimento de um sistema fluvio-lacustre com influência marinha pode estar associado à separação das áreas de endemismo Imeri e Napo/Inambari, mas o que dizer da área de endemismo Rio Negro? Este caso talvez não esteja associado aos eventos geológicos do Mioceno. Atualmente, a área Rio Negro é contígua com a área Imeri. Entretanto, um imenso bloco de vegetações mais abertas de campina e campinarana se estende entre o médio e o alto Rio Negro (Ibge 1997). Estes tipos de vegetações podem ter ocupado uma extensão ainda maior durante o Pleistoceno. Paleodunas encontradas entre os rio Branco e Negro foram depositadas durante as fases mais secas do Holoceno (CARneiro Filho et al. 2002). A formação destas dunas de areia aparentemente foi intermediada por ventos regionais (deposição eólica) sugerindo uma paisagem dominada por vegetações abertas no período de deposição (CARnEIro FILHo et al. 2002). Ainda precisa ser melhor estabelecido se as vegetações predominantemente abertas encontradas no médio Rio Negro representam uma barreira ecológica para a distribuição de aves. Se este for o caso, a avifauna da área de endemismo Rio Negro talvez tenha se isolado do Imeri durante períodos de tempo mais recentes do Quaternário.

Neste ponto é importante lembrar uma das mais controvertidas questões sobre os paleo-ambientes da Amazônia que postula períodos de aridez durante o Pleistoceno quando a vegetação florestal da região foi substituída por savanas abertas. Esta controvérsia é a base para a mais famosa hipótese para explicar a diversificação da avifauna na Amazônia - a teoria dos refúgios de HAFFer (1969). Esta teoria postula períodos secos durante o Pleistoceno onde a paisagem amazônica foi dominada por savana e as florestas se restringiram às porções mais úmidas da bacia (HafFer 1969). O isolamento das populações de aves nestes blocos de florestas (denominados refúgios) iniciou um processo de diferenciação alopátrica (HAFFer 1969). Alguns pesquisadores afirmam que as evidências geológicas, palinológicas e geomorfológicas não são consistentes com uma savanização extensa da Amazônia durante o Pleistoceno (Salo 1987, Colinvaux et al. 2001). Outros admitem que regiões atualmente cobertas por florestas podem ter sido ocupadas por vegetações mais abertas do tipo savana ou por florestas mais secas distribuídas em matas de galeria (Pennington et al. 2000, Haffer 2001).

O debate sobre como foi a paisagem que ocupou a bacia amazônica durante o Quaternário está longe de ser finalizado e não é objetivo deste estudo oferecer evidências diretas para esta questão. Entretanto, um período mais seco onde vegetações mais abertas dominaram o médio Rio Negro parece consistente com o isolamento entre as porção baixa (área de endemismo Rio Negro) e alta (área de endemismo Imeri) do Rio Negro.

Revista Brasileira de Zoologia 24 (4): 919-940, dezembro 2007 
Este cenário contrasta diretamente com as proposições de divergência mais antiga das aves amazônicas discutidas acima. Entretanto, estudos de diferenciação genética em araçaris (Pteroglossus Illiger, 1811 - Ramphastidae) sugerem eventos de divergência recentes que podem ter ocorrido durante o Pleistoceno (HACKET \& LeHN 1997). Um dos táxons que dão suporte à area de endemismo Rio Negro é um araçari do mesmo gênero estudado por HACKet \& LeHN (1997) - Pteroglossu azara azara Vieillot, 1819. Os estudos moleculares sugerem que aves amazônicas de linhagens antigas e recentes convivem lado a lado na mesma avifauna.

A história de diversificação da avifauna dos setores noroeste e oeste da bacia Amazônica pode ter sido influenciada por eventos antigos (transgressões marinhas do Mioceno) e eventos recentes (vegetações abertas do Quaternário). Esta linha de argumentação sobre os processos que levaram à diferenciação das espécies de aves precisa ser testada através de revisões taxonômicas mais detalhadas, estabelecimento de filogenias explícitas e análises filogeográficas dos táxons envolvidos (Moritz et al. 2000, Arbogast \& Kenagy 2001).

\section{AGRADECIMENTOS}

Os estudos de aves no Parque Nacional do Jaú têm sido financiados pela Fundação Vitória Amazônica (FVA), European Commission, WWF-Brasil, Gordon \& Betty Moore Foundation e CNPq. Este estudo também recebeu auxílio financeiro da CAPES através de um bolsa de doutorado e WWF-Brasil através do programa Natureza \& Sociedade. Teresa C. Ávila-Pires, Maria L. Marceliano, Maria A. dos S. Alves, Alexandre Aleixo e Luiz P. Gonzaga deram importantes contribuições a este estudo. Fátima Lima, Dionísio Pimentel, Ramiro, Fernando Novaes (in memorian) e Maria Luiza ajudaram no uso da coleção de aves do Museu Paraense Emílio Goeldi (MPEG). Mario CohnHaft, Alexandre Aleixo e José M.C. da Silva contribuiram com importantes "insights" para este estudo. Bret Whitney gentilmente providenciou a lista de aves de Quebrada Sucusari utilizada nas análises. Simone Iwanaga, Roni von Lira, Marcelo Moreira e Ricardo Almeida ajudaram na produção de mapas e edição das figuras.

\section{REFERÊNCIAS BIBLIOGRÁFICAS}

Aleixo, A. 2002. Molecular systematics and the role of the "várzea-terra firme" ecotone in the diversification of Xiphorhynchus woodcreepers (Aves: Dendrocolaptidae). Auk 119: 621-640.

Aleixo, A. \& B. Whitney. 2002. Dendroplex (= Xiphorhynchus) necopinus Zimmer, 1934 (Dendrocolaptidae) is a junior synomym of Dendrornis kienerii (= Xiphorhynchus picus kienerii) Des Murs, 1855. Auk 119: 520-523.

Aleixo, A.; B.M. Whitney \& D. Oren. 2000. Range extensions of birds in southeastern Amazonia. Wilson Bulletin 112: 137142.

Revista Brasileira de Zoologia 24 (4): 919-940, dezembro 2007
Alonso, J.A. \& B.M. Whitney. 2001. A new Zimmerius tyrannulet (Aves: Tyrannidae) from white sand forests of northern Amazonian Peru. Wilson Bulletin 113: 1-9.

Alonso, J.A. \& B.M. Whitney. 2003. New distributional records of birds from white-sand forests of the northern peruvian Amazon, with implications for biogeography of northern South America. Condor 105: 552-566.

Amorim, D.S. \& M.R. PIREs. 1999. Neotropical biogeography and a method for maximum biodiversity estimation, p. 183-219. In: C.E.M Bicudo \& N.A. Menezes (Eds). Biodiversity in Brazil: a first approach. Proceedings of the Workshop Methods for the Assessment of Biodiversity in Plants and Animals. São Paulo, Instituto de Botânica, CNPq, 326p.

Anderson, A. 1981. White-sand vegetation of Brazilian Amazonia. Biotropica 13: 199-210.

Arbogast, B.S. \& G.J. Kenagy. 2001. Comparative phylogeography as an integrative approach to historical biogeography. Journal of Biogeography 28: 819-825.

ÁvILA-PIRES, T.C.S. 1995. Lizards of Brazilian Amazonia (Reptilia: Squamata). Zoologische Verhandelingen Nationaal Natuurhistorisch Museum, 709p.

Ayres, M. \& T.H. Clutton-Brock. 1992. River boundaries and species range size in Amazonian primates. American Naturalist 140: 531-537.

BALL, I.R. 1975. Nature and formulation of biogeographical hypotheses. Systematic Zoology 24: 407-430.

Bates, J.M. 2001. Avian diversification in Amazonia: evidence for historical complexity and a vicariance model for a basic diversification pattern, p. 119-138. In: I.C.G. VIEIRA; J.M.C. DA Silva; D.C. Oren \& M.A. D'InCAo (Eds). Diversidade cultural e biológica da Amazônia. Belém, Museu Paraense Emílio Goeldi, 421p.

Bates, J.M.; S. Hackett \& J. Cracraft. 1998. Area-relationships in the Neotropical lowlands: an hypothesis based on raw distributions of Passerine birds. Journal of Biogeography 25: 783-793.

Bates, J.M. \& T. Demos. 2001. Do we need to devalue Amazonia and other large tropical forests? Diversity and Distributions 7: 249-255.

Bierregaard, R.O.; M. Cohn-Haft \& D. Stotz. 1997. Cryptic biodiversity: an overlooked species and new subspecies of antbird (Aves: Formicariidae) with a revision of Cercomacra tyrannina in north-eastern South America. Ornithological Monographs 48: 111-128.

Bisconti, M.; W. Landini; G. Bianucci; G. Cantalamessa; G. Carnevale; L. Ragaini \& G. Valeri. 2001. Biogeography relationships of the Galapagos terrestrial biota: parsimony analysis of endemicity based on reptiles, land birds and Scalesia land plants. Journal of Biogeography 28: 495-510.

Borges, S.H. \& A.M.P. Carvalhaes. 2000. Bird species of black water inundation forests in the Jaú National Park (Amazonas State, Brazil): their contribution to regional species richness. Biodiversity and Conservation 9: 201-214. 
Borges, S.H.; M. Cohn-Haft; A.M.P. Carvalhaes; L.M. Henriques; J.F. Pacheco \& A. WhitTaker. 2001. Birds of Jaú National Park, Brazilian Amazon: species check-list, biogeography and conservation. Ornitologia Neotropical 12: 109-140.

Borges, S.H. 2004. Species poor but distinct: bird assemblages in white sand vegetation in Jaú National Park, Amazonian Brazil. Íbis 146: 114-124.

Borges, S.H. \& R.A. Almeida. 2001. First Brazilian record of the Yapacana Antbird (Myrmeciza disjuncta, Thamnophilidae) with additional notes on its natural history. Ararajuba 9 : 163-165.

Bremer, K. 1994. Branch support and tree stability. Cladistics 10: 295-304.

Brooks, D.R. \& M.G.P. van Veller. 2003. Critique of parsimony analysis of endemicity as a method of historical biogeography. Journal of Biogeography 30: 819-825.

Brumfield, R.T.; D.L. Swofford \& M.J. Braun. 1997. Evolutionary relationships among the potoos (Nyctibiidae) based on isozymes. Ornithological Monographs 48: 129-146.

Bush, M. 1994. Amazonian speciation: a necessarily complex model. Journal of Biogeography 21: 5-17.

Capparela, A.P.; G.H. Rosenberg \& S. Cardiff. 1997. A new subspecies of Percnostola rufifrons (Formicariidae) from northeastern Amazonian Peru, with a revision of the rufifrons complex. Ornithological Monographs 48: 165-170.

Carneiro-Filho, A.; D. Schwartz; S.H. Tatumi \& T. Rosique. 2002. Amazonian paleodunes provide evidence for drier climate phases during the Late Pleistocene-Holocene. Quaternary Research 58: 205-209.

Cohn-Haft, M.; A.Whittaker \& P.C. Stouffer. 1997. A new look at the "species-poor" central Amazon: the avifauna north of Manaus, Brazil. Ornithological Monographs 48: 205235.

Colinvaux, P.A.; G. Irion; M.E. Rasanen; M.B.Bush \& J.A.S.N. MelLo. 2001. A paradigm to be discarded: geological and paleoecological data falsify the Haffer \& Prance refuge hypothesis of Amazonian speciation. Amazoniana XVI: 609-646.

Costa, L.P.; Y.L.R. Leite; G. Fonseca \& M.T. Fonseca. 2000. Biogeography of South America forest mammals: endemism and diversity in the Atlantic Forest. Biotropica 32: 872-881.

Cracraft, J. 1983. Species concepts and speciation analysis. Current Ornithology 1: 159-187.

Cracraft, J. 1985. Historical biogeography and patterns of differentiation within the South American avifauna: areas of endemisms. Ornithological Monographs 36: 49-84.

Cracraft, J. 1988. Deep-history biogeography: retrieving the historical pattern of evolving continental biotas. Systematic Zoology 37: 221-236.

Cracraft, J. 1994. Species diversity, biogeography, and the evolution of biotas. American Zoology 34: 33-47.

Cracraft, J. \& R. Prum. 1988. Patterns and processes of diversification: speciation and historical congruence in some neo- tropical birds. Evolution 42: 603-620.

del Hoyo, J.; A. Elliot \& J. Sargatal. 1992. Handbook of the Birds of the World. Ostrich to Ducks. Linx Edicions, Barcelona, vol. 1, 696p.

del Hoyo, J.; A. Elliot \& J. Sargatal. 1993. Handbook of the Birds of the World. New World Vultures to Guineafowl. Barcelona, Linx Edicions, vol. 2, 638p.

del Hoyo, J.; A. Elliot \& J. Sargatal. 1996. Handbook of the Birds of the World. Hoatzin to Auks. Barcelona, Linx Edicions, vol. 3, 821p.

del Hoyo, J.; A. Elliot \& J. Sargatal. 1997. Handbook of the Birds of the World. Sandgrouse to Cuckoos. Barcelona, Linx Edicions, vol, 4, 679p.

del Hoyo, J.; A. Elliot \& J. Sargatal. 1999. Handbook of the Birds of the World. Barn-Owls to Hummingbirds. Barcelona, Linx Edicions, vol. 5, 759p.

del Hoyo, J.; A. Elliot \& J. Sargatal. 2001. Handbook of the Birds of the World. Mousebirds to Hornbills. Barcelona, Linx Edicions, vol. 6, 589p.

del Hoyo, J.; A. Elliot \& J. Sargatal. 2002. Handbook of the Birds of the World. Jacamars to Woodpeckers. Barcelona, Linx Edicions, vol. 7, 613p.

del Hoyo, J.; A. Elliot \& J. Sargatal. 2003. Handbook of the Birds of the World. Broadbills to Tapaculos. Barcelona, Linx Edicions, vol. 8, 845p.

del Hoyo, J.; A. Elliot \& J. Sargatal. 2004. Handbook of the Birds of the World. Cotingas to Wagtails. Barcelona, Linx Edicions, vol. 9, 863p.

Eisenmann, E. \& L. Short. 1982. Systematics of the avian genus Emberizoides (Emberizidae). American Museum Novitates 2740: $1-21$.

ENDLER, J. 1982. Pleistocene forest refuges: fact or fancy, p. 179200. In: G.T. Prance (Ed.). Biological diversification in the tropics. New York, Columbia University Press, 714p.

Ferreira, L.V. 1997. Effects of the duration of flooding on species richness and floristics composition in three hectares in Jaú National Park in floodplain forest in Central Amazonia. Biodiversity and Conservation 6: 1353-1363.

Ferreira, L.V. \& G.T. Prance. 1998. Species richness and floristic composition in four hectares in the Jaú National Park in upland forests in Central Amazonia. Biodiversity and Conservation 7: 1349-1364.

Fernandes, M.E.B.; J.M.C. DA Silva \& J.S. Silva Jr. 1995. The monkeys of the islands of the Amazon estuary, Brazil: a biogeographic analysis. Mammalia 59: 213-221.

FriedmanN, H. 1948. Birds collected by the National Geographic Society's expeditions to northern Brazil and southern Venezuela. Proceedings of the Unites States National Museum 97: 373-570.

Fva/Iвама. 1998. Plano de manejo do Parque Nacional do Jaú. Fundação Vitória Amazônica, IBAMA, Manaus, 258p.

Gárcia-Barros, E.; P. Gurrea; M.J. Luciáñez; J.M. Cano; M.L. Munguira; J.C.Moreno; H. Sainz; M.J. Sanz \& J.C. Simón. 2002. 
Parsimony analysis of endemicity and its application to animal and plant geographical distributions in the IberoBalearic region (western Mediterranean). Journal of Biogeography 29: 109-124.

Grau, E.T; S.L. Pereira; L.F. Silveira; E. Höfling \& A. Wajntal. 2005. Molecular phylogenetic and biogeography of Neotropical piping guans (Aves: Galliformes): Pipile Bonaparte, 1856 is synonym of Aburria Reichenbach, 1853. Molecular Phylogenetics and Evolution 35: 637-645.

Hacketт, S. 1993. Phylogenetic and biogeography relationships in the neotropical genus Gymnopithys (Formicariidae). Wilson Bulletin 105: 301-315.

Наскетт, S. 1996. Molecular phylogenetics and biogeography of tanagers in the genus Ramphocelus (Aves). Molecular Phylogenetics and Evolution 5: 368-382.

Hackett, S. \& C.A. Lehn. 1997. Lack of genetic divergence in a genus (Pteroglossus) of Neotropical birds: the connection between life-history characteristics and levels of genetic divergence. Ornithological Monographs 48: 267-280.

Haffer, J. 1969. Speciation in Amazonian forest birds. Science 165: 131-137.

Haffer, J. 1974. Avian speciation in tropical South America. Publications of the Nuttal Ornithological Club 14: 1-390.

HAfFer, J. 1978. Distribution of Amazon forest birds. Bonner Zoologische Beitrage 29: 38-78.

Haffer, J. 1985. Avian zoogeography of the neotropical lowlands. Ornithological Monographs 36: 113-145.

Haffer, J. \& J.W. Fitzpatrick. 1985. Geographic variation in some amazonian forest birds. Ornithological Monographs 36: 147-168.

Haffer, J. 1992. On the "river effect" in some forest birds of southern Amazonia. Boletim do Museu Paraense Emílio Goeldi, Série Zoologia, 8: 217-245.

Haffer, J. 2001. Hypotheses to explain the origin of species in Amazonia, p. 45-118. In: I.C.G. Vieira; J.M.C. DA Silva; D.C. OREN \& M.A. D'InCAO (Eds). Diversidade cultural e biológica da Amazônia. Belém, Museu Paraense Emílio Goeldi, $421 \mathrm{p}$.

Hilty, S.L. 2003. Birds of Venezuela. Princeton, Princeton University Press, $2^{\text {nd }}$ ed., 878 p.

Hinkelmann, C. 1996. Systematics and geographic variation in long-tailed hermit hummingbirds, the Phaethornis superciliosus-malaris-longirostris species group (Trochilidae), with notes on their biogeography. Ornitologia Neotropical 7: 119-148.

Hoorn, C. 1993. Marine incursions and the influence of Andes tectonics on the Miocene depositional history of northwestern Amazonia: results of a palynostratigraphic study. Palaegeography, Palaeoclimatology, Palaeoecology 105: 267-309.

Hoorn, C. 1994a. Fluvial palaeoenvironments in the intracratonic Amazonas Basin (Early Miocene - early Middle Miocene, Colombia). Palaegeography, Palaeoclimatology,
Palaeoecology 109: 1-57.

Hoorn, C. 1994b. An environmental reconstruction of the paleoamazon River system (Middle-Late Miocene, NW Amazonia). Palaegeography, Palaeoclimatology, Palaeoecology 112: 187-238.

Hu, DA-Shin; L. Joseph \& D. Agro. 2000. Distribution, variation, and taxonomy of Topaza hummingbirds (Aves: Trochilidae). Ornitologia Neotropical 11: 123-142.

Humphries, C.J. 1989. Any advance in assumption 2? Journal of Biogeography 16: 101-102.

IBGE. Diagnóstico ambiental da Amazonia Legal. Rio de Janeiro, IBGE [1997, CD-ROM].

Isler, M.; J.A. Alonso; P.R. Isler \& B.M Whitney. 2001. A new species of Percnostola antbird (Passeriformes: Thamnophilidae) from Amazonian Peru, and an analysis of species limits within Percnostola rufifrons. Wilson Bulletin 113:164-176.

IsLER, M.L.\& P.R. ISLER. 1987. The tanagers - natural history, distribution, and identification. Washington, Smithsonian Institution Press, 406p.

Isler, M.L.; P.R. IsLer \& B. Whitney. 1999. Species limits in antbirds (Passeriformes; Thamnophilidae): the Myrmotherula surinamensis complex. Auk 116: 83-96.

Lovejoy, N.R.; E. Bermingham \& A.P. Martin. 1998. Marine incursions into South America. Nature 396: 421-422.

Lundberg, J.G.; L.G. Marshall; J. Guerrero; B. Horton; M.C. Malabarba \& F. Wesselingh. 1998. The stage for neotropical fish diversification: a history of tropical South America rivers, p. 13-48. In: L.R. Malabarba; R.E. Reis; R.P. Vari; C.A.S. LuCENA \& Z.M.S. LuCENA (Eds). Phylogeny and Classification of Neotropical Fishes. Porto Alegre, EDIPUCRS, 603p.

LyNCH, J.D. 1989. The gauge of speciation, p. 527-533. In: D. OtTe \& J.A. EndLer (Eds). Speciation and its consequences. Sunderlands, Sinauer, 679p.

Marantz, C. 1997. Geographic variation of plumage patterns in the woodcreeper genus Dendrocolaptes (Dendrocolaptidae). Ornithological Monographs 48: 399-430.

Marroig, G. \& R. Cerqueira 1997. Plio-Pleistocene South American history and the Amazon laggon hypothesis: a piece in the puzzle of amazonian diversification. Journal of Comparative Biology 2: 103-119.

Martuscelli, P. \& C. Yamashita. 1997. Rediscovery of the Whitecheeked Parrot Amazona kawalli (Grantsau and Camargo, 1989), with notes on its ecology, distribution, and taxonomy. Ararajuba 5: 97-113.

Mccune, B. \& J. Grace. 2002. Analysis of ecological communities. Gleneden Beach, MjM Software Design, 304p.

Moritz, C.; J.L. Patton; C.J. Schneider \& T.B. Smith. 2000. Diversification of rainforest faunas: an integrated molecular approach. Annual Review Ecology, Evolution and Systematic 31: 533-563.

Morner, N.; D. Rosseti \& P.M. Toledo. 2001. The Amazon rainforest only 6-5 millions years old, p. 3-18. In: I.C.G. Vieira; J.M.C. da Silva; D.C. Oren \& M.A. D’Incao (Eds). Di- 
versidade cultural e biológica da Amazônia. Belém, Museu Paraense Emílio Goeldi, 421p.

Morrone, J.J. 1994. On the identification of areas of endemism. Systematic Biology 43: 438-441.

Morrone, J.J. \& J.V. Crisci. 1995. Historical biogeography: introduction to methods. Annual Review Ecology, Evolution and Systematic 26: 373-401.

Naka, L.N; M. Cohn-Haft; F. Mallet-Rodrigues; M.P.D. Santos \& M.F. Torres. 2006. The avifauna of the Brazilian state of Roraima: bird distribution and biogeography in the Rio Branco basin. Revista Brasileira de Ornitologia 14: 197-238.

NiHeI, S.S. 2006. Misconceptions about parsimony analysis of endemicity. Journal of Biogeography 33: 2099-2106.

Nores, M. 1999. An alternative hypothesis for the origin of Amazonian bird diversity. Journal of Biogeography 26: 475485.

NorgaARd-Olesen, E. 1973. Tanagers. Denmark, Skibby Books, vol. 1, 255p.

Norgaard-Olesen, E. 1974. Tanagers. Denmark, Skibby Books, vol. 2, 216p.

Novaes, F.C. 1994. Aves da floresta de igapó, Rio Negro (Estado do Amazonas), Brasil. Boletim do Museu Paraense Emílio, Série Zoologia, 10: 155-167.

Novaes, F.C. \& M.F.C. Lima. 1991. As aves do Rio Peixoto de Azevedo, Mato Grosso, Brasil. Revista Brasileira de Zoologia $7:$ 351-381.

Novaes, F.C. \& M.F.C. Lima, 1998. Aves da Grande Belém, municípios de Belém e Ananindeua, Pará. Belém, Museu Paraense Emílio Goeldi, 415p.

NutTAL, C.P. 1990. A review of the Tertiary non marine molluscans faunas of the Pebasian and other inland basins of northwestern South America. Bulletin British Museum of Natural History, Geology, 45: 165-371.

Oren, D. 2001. Biogeografia e conservação de aves na região amazônica, p. 268-286. In: J.P. CApobianco; A. Veríssimo; A. Moreira; D. Sawyer; I dos Santos \& L.P Pinto (Eds). Biodiversidade na Amazônia brasileira: avaliação e ações prioritárias para a conservação, uso sustentável e repartição de benefícios. São Paulo, Estação da Liberdade, Instituto Socioambiental, 540p.

Oren, D.C. \& H.G. Albuquerque. 1991. Priority areas for new avian collections in Brazilian Amazonia. Goeldiana, Zoologia, 6: 1-11.

Patton, J. \& M.N. Silva. 2001. Molecular phylogenetics and the diversification of Amazonian mammals. p. 139-166. In: I.C.G. Vieira; J.M.C. DA Silva; D.C. Oren \& M.A. D’InCao (Eds). Diversidade cultural e biológica da Amazônia. Belém, Museu Paraense Emílio Goeldi, 421p.

Pennington, R.T.; D.E. Prado \& C. Pendry. 2000. Neotropical seasonally dry forests and Quaternary vegetation changes. Journal of Biogeography 27: 261-273.

Pereira, S.L. \& A.L. BAKER. 2004. Vicariant speciation of currasows (Aves, Cracidae): a hypothesis based on mitochondrial DNA phylogeny. Auk 121: 682-694.

Peres, C. \& A. Whittaker. 1991. Annotated checklist of the bird species of the upper Rio Urucu, Amazonas, Brazil. Bulletin of the British Ornithologists' Club 111: 156-171.

PINTO, O. 1944. Catálogo de aves do Brasil. São Paulo, Secretária da Agricultura, Indústria e Comércio, parte 2, 700p.

PINTO, O. 1978. Novo catálogo das aves do Brasil. São Paulo, Brasil, parte 1, 446p.

Pinto, O. \& E.A. Camargo. 1957. Sôbre uma coleção de aves da região de Cachimbo (Sul do Estado do Pará). Papéis Avulsos do Departamento de Zoologia 13: 51-69.

Pires, J.M. \& G.T. Prance. 1985. The vegetation types of Brazilian Amazon, p. 109-145. In: G.T. Prance \& T.E. Lovejoy (Eds). Key environment - Amazonia. New York, Pergamon Press, 442p.

Poleto, F. \& A. Aleixo. 2005. Implicações biogeográficas de novos registros ornitológicos em um enclave de vegetação de campina no sudoeste da Amazônia brasileira. Revista Brasileira de Zoologia 22: 1196-1200.

Prum, R.; J.D. Kaplan \& J.E. Pierson. 1996. Display behavior and natural history of the Yellow-crowned Manakin (Heterocercus flavivertex: Pipridae). Condor 98: 722-735.

Rasänen, M.; A.M. Linna; J.C.R. Santos \& F.R. Negri. 1995. Late Miocene tidal deposits in the Amazon foreland basin. Science 269: 386-390.

ReMSEN, J.V. 1994. Use and misuse of bird lists in community ecology and conservation. Auk 111: 225-227.

Ribas, C.C., R. Gaban-Lima, C.Y. Miyaki \& J. Cracraft. 2005. Historical biogeography and diversification within the Neotropical parrot genus Pionopsitta (Aves: Psittacidae). Journal of Biogeography 32: 1409-1427.

Ridgely, R.S. \& G. Tudor. 1989. The birds of South America. The Oscines Passerines. Austin, University of Texas, vol. 1, $596 \mathrm{p}$.

Ridgely, R.S. \& G. Tudor. 1994. The birds of South America. The Suboscines Passerines. Austin, University of Texas, vol. 2, 940p.

Robbins, M.B. \& T.A. Parker. 1997. Voice and taxonomy of Caprimulgus (rufus) otiosus (Caprimulgidae), with a reevaluation of Caprimulgus rufus subspecies. Ornithological Monographs 48: 601-608.

RoN, S.R. 2000. Biogeographical area relationships of lowland Neotropical rainforest based on raw distributions of vertebrates groups. Biological Journal of the Linnean Society 71: 379-402.

Rosen, B.R. 1995a. Biogeographic patterns: a perceptual overview, p. 21-55. In: A. Myers \& P. GILler (Eds). Analytical biogeography: an integrated approach to the study of animal and plant distribution. Londres, Chapman \& Hall, $584 \mathrm{p}$.

Rosen, B.R. 1995b. From fossils to earth history: applied historical biogeography, p. 437-481. In: A. Myers \& P. Giller (Eds). Analytical biogeography: an integrated approach to the 
study of animal and plant distribution. Londres, Chapman \& Hall, 584p.

Rylands, A.B. 1991. The status of conservation areas in the Brazilian Amazon. Washington, World Wildlife Fund, The Conservation Foundation, 146p.

SALO, J. 1987. Pleistocene forest refuges in the Amazon: evaluation of the biostratigraphical, lithostratigraphical and geomorphological data. Annales Zoologici Fennici 24: 203-211.

SANTOS, C.M. 2005. Parsimony analysis of endemicity: time for an epitaph? Journal of Biogeography 32: 1281-1286.

Sick, H. 1967. Rios e enchentes na Amazônia como obstáculo para a avifauna. Manaus, Atas Simpósio Sobre a Biota amazônica 5: 495-520.

SICK, H. 1997. Ornitologia brasileira. Rio de Janeiro, Nova Fronteira, 912p.

SiLva, J.M.C. \& D.C. Oren. 1996. Application of parsimony analysis of endemicity in Amazonian biogeography: an example with primates. Biological Journal of the Linnean Society 59: 427437.

Silva, J.M.C.; F.C. Novaes \& D.C. Oren. 2002 Differentiation of Xiphocolaptes (Dendrocolaptidae) across the river Xingu, Brazilian Amazonia: recognition of a new phylogenetic species and biogeographical implications. Bulletin of the British Ornithologists' Club 122: 185-194.

SiLva, M.N.F. \& J.L. PATTON. 1998. Molecular phylogeography and the evolution and conservation of Amazonian mammals. Molecular Ecology 7: 475-486.

Smith, T.B.; R.K.Wayne; D.J. Girman \& M.W. Bruford. 1997. A role of ecotones in generating rainforest biodiversity. Science 276: $1855-1857$.

Stotz, D.; S.M. Lanyon; T.S. Schulenberg; D.E. Willard; A.T. Peterson \& J. Fitzpatrick. 1997. An avifaunal survey of two tropical forest localities on the middle Rio Jiparaná, Rondônia, Brazil. Ornithological Monographs 48: 763-782.

Swofford, D.L. 1991. PAUP*: Phylogenetic Analysis Using Parsimony. Illinois, Center for Biodiversity of the Illinois Natural History Survey.
Tuomisto, H. \& K. Ruokolainen. 1997. The role of ecological knowledge in explaining biogeography and biodiversity in Amazonia. Biodiversity and Conservation 6: 347-357.

VicentinI, A. 2004. A vegetação ao longo de um gradiente edáfico no Parque Nacional do Jaú, p. 105-131. In: S.H. Borges; S. Iwanaga; C.C. Durigan \& M.R. Pinheiro (Eds). Janelas para a biodiversidade no Parque Nacional do Jaú: uma estratégia para o estudo da biodiversidade na Amazônia. Manaus, Fundação Vitória Amazônica, WWF-Brasil, USAID, 275p.

Wallace, A.R. 1852. On the monkeys of the Amazon. Proccedings of Zoological Society of London 1852: 107-110.

Wetterberger, G.B.; M.T. Jorge-Pádua; C.S. Castro \& J.M.C. VasCONCELOS. 1976. Uma análise de prioridades em conservação da natureza na Amazônia. Brasília, Projeto de Desenvolvimento e Pesquisa Florestal, PNUD, FAO, IBDF, BRA 45, Série Técnica, vol. 8, 63p.

Whitney, B. \& J.A. Alonso. 1998. A new Herpsilochmus antwren (Aves: Thamnophilidae) from northern Amazon Peru and adjacent Ecuador: the role of edaphic heterogeneity of terra firme forest. Auk 115: 559-576.

Zimmer, K. 1997. Species limits in Cranioleuca vulpina. Ornithological Monographs 48: 849-864.

Zimmer, K. 1999. Behavior and vocalizations of the Caura and the Yapacana Antbird. Wilson Bulletin 111: 195-209.

ZimMER, K. \& S. HiLty. 1997. Avifauna of a locality in the upper Orinoco drainage of Amazonas, Venezuela. Ornithological Monographs 48: 865-886.

Zimmer, K.; T.A. Parker; M. Isler \& P. Isler. 1997. Survey of a southern Amazonian avifauna: the Alta Floresta region, Mato Grosso, Brazil. Ornithological Monographs 48: 887-918.

Zimmer, K.J. \& A. WhitTaKer. 2000. Species limits in Pale-tipped tyrannulets (Inezia: Tyrannidae). Wilson Bulletin 112: 5166.

Recebido em 30.XI.2006; aceito em 13.XI.2007.

Anexo I. Lista das espécies de aves registradas no Parque Nacional do Jaú examinada neste estudo. Os números sobrescritos ao lado de algumas espécies referem-se às notas taxonômicas e biogeograficas encontradas no final da lista. As letras entre parênteses são as categorias de distribuição geográfica descritas na tabela I e os números referem-se aos registros dos exemplares consultados na coleção de aves do Museu Paraense Emílio Goeldi (MPEG). A sequência das espécies segue Sıck (1997) para as famílias de aves não Passeriforme e Ridgely \& TUdor $(1989,1998)$ para famílias Passeriformes. A nomenclatura das espécies foi atualizada seguindo as recomendações do Comitê Brasileiro de Registros Ornitológicos (www.cbro.org.br).

Tinamidae: Tinamus major serratus (Spix, 1825) (b, 31613; 29937), Crypturellus cinereus ${ }^{1}$ (Gmelin, 1789) (a), Crypturellus soui soui (hermann, 1783) (c, 31614), Crypturellus undulatus yapura (Spix, 1825) (d), Crypturellus variegatus² (Gmelin, 1789) (a). Accipitridae: Leptodon cayanensis cayanensis ${ }^{3}$ (Latham, 1790) (a), Ictinia plumbea (Gmelin, 1788) (a), Accipiter bicolor bicolor (Vieillot, 1817) (a), Buteo nitidus nitidus (Latham, 1790) (a), Rupornis magnirostris magnirostris (Gmelin, 1788) (a), Busarellus nigricollis nigricollis (Latham, 1790) (a), Buteogallus urubitinga urubitinga (Gmelin, 1788) (a), Harpia harpyja (Linnaeus, 1758) (a), Spizaetus ornatus ornatus (Daudin, 1800) (a), Spizaetus tyrannus serus (Friedmann, 1950) (a), Geranospiza caerulescens caerulescens (Vielliot, 1817) (a). Falconidae: Herpetotheres cachinnans cachinnans (Linnaeus, 1758) (a), Micrastur semitorquatus semitorquatus (Vielliot, 1817) (a), Micrastur ruficollis concentricus ${ }^{4}$ (Lesson, 1830) (c), Micrastur gilvicollis ${ }^{5}$ (Vieillot, 1817) (a, 50523; 42442; 52946), Micrastur mirandollei6 (Schlegel, 1862) (a), Daptrius

Revista Brasileira de Zoologia 24 (4): 919-940, dezembro 2007 
ater (Vieillot, 1816) (a), Ibycter americanus7 (Boddaert, 1783) (a), Milvago chimachima cordatus (Bangs \& Penard, 1918) (c), Falco rufigularis rufigularis (Daudin, 1800) (a). Cracidae: Penelope jacquacu orienticola (Todd, 1932) (b, 118), Pipile cumanensis cumanensis (Jacquin, 1784) (c), Mitu tuberosum ${ }^{8}$ (Spix, 1825) (a), Nothocrax urumutum (Spix, 1825) (d). Phasianidae: Odontophorus gujanensis buckleyi (Chubb, 1919) (h, 50524; 31672). Opisthocomidae: Opisthocomus hoazin (Sclater, 1880) (a). Psophiidae: Psophia crepitans ochroptera $^{10}$ (Pelzen, 1857) (g). Rallidae: Aramides cajanea cajanea (P.L.S. Müller, 1776) (a), Laterallus melanophaius oenops (P.L. Sclater \& Salvin, 1880) (a). Columbidae: Patagioenas speciosa (Gmelin, 1789) (a), Patagioenas cayennensis cayennensis (Bonnaterre, 1792) (c, 17472), Patagioenas subvinacea purpureotincta (Ridgway, 1888) (c, 42446-47), Patagioenas plumbea wallacei (Chubb, 1917) (c, 52497), Columbina passerina griseola (Spix, 1825) (a, 27847-51), Columbina talpacoti talpacoti (Temminck, 1811) (a), Leptotila verreauxi brasiliensis (Bonaparte, 1856) (a), Leptotila rufaxilla dubusi (Bonaparte, 1825) (d, 42448; 27843-45), Geotrygon montana montana (Linnaeus, 1758) (a). Psittacidae: Ara ararauna (Linnaeus, 1758) (a), Ara macao macao (Linnaeus, 1758) (a), Ara chloropterus (Gray, 1859) (a), Orthopsittaca manilata (Boddaert, 1783) (a), Aratinga leucophthalma callogenys (Salvadori, 1891) (d), Aratinga pertinax chrysogenys (Massena \& Souancé, 1854) (b), Pyrrhura melanura melanura ${ }^{11}$ (Spix, 1824) (e, 42454; 17457), Brotogeris chrysoptera tenuifrons (Friedmann, 1945) (b, 29985), Touit purpuratus viridiceps ${ }^{12}$ Chapman, 1929 (d), Touit huetii (Temminck, 1830) (a), Pionites melanocephalus melanocephalus (Linnaeus, 1758) (c), Pionopsitta barrabandi barrabandi (Kuhl, 1820) (e), Pionus menstruus menstruus (Linnaeus, 1766) (a), Pionus fuscus fuscus ${ }^{13}$ (Statius Muller, 1776) (a), Amazona autumnalis diadema (Spix, 1824) (a), Amazona festiva festiva (Linnaeus, 1758) (a, 42460), Amazona amazonica $^{14}$ (Linnaeus, 1766) (a), Amazona farinosa farinosa (Boddaert, 1783) (a), Amazona kawalli15 Grantsau \& Camargo, 1989 (a), Deroptyus accipitrinus accipitrinus (Linnaeus, 1758) (c). Cuculidae: Piaya cayana cayana ${ }^{16}$ (Linnaeus, 1766) (c, 17535), Piaya melanogaster melanogaster (Vieillot, 1817) (a), Coccycua minuta minuta (Vieillot, 1817) (a, 42461), Crotophaga ani Linnaeus, 1758 (a), Crotophaga major Gmelin, 1788 (a), Tapera naevia naevia (Linnaeus, 1766) (a). Tytonidae: Tyto alba hellmayri Griscom \& Greenway, 1937 (c). Strigidae: Megascops choliba crucigerus (Spix, 1824) (a, 27841), Megascops watsonii usta (P.L. Sclater, 1859) (d, 52948; 42462), Lophostrix cristata cristata (Daudin, 1800) (a), Pulsatrix perspicillata perspicillata (Latham, 1790) (a), Glaucidium brasilianum ucayale Chapman, 1929 (f), Strix huhula huhula Daudin, 1800 (a), Asio stygius stygius (Wagler, 1832) (a). Nyctibiidae: Nyctibius grandis grandis (J. F. Gmelin, 1789) (a), Nyctibius aethereus longicaudatus (Spix, 1825) (a), Nyctibius griseus griseus (J. F. Gmelin, 1789) (a, 50525), Nyctibius leucopterus ${ }^{17}$ (Wied, 1821) (a, 50526-27; 46870), Nyctibius bracteatus Gould, 1846 (a, 52954). Caprimulgidae: Lurocalis semitorquatus semitorquatus (J. F. Gmelin, 1789) (c, 52956; 30008-09), Nyctidromus albicollis albicollis (Gmelin, 1789) (a), Caprimulgus rufus rufus ${ }^{18}$ Boddaert, 1783 (a), Caprimulgus cayennensis cayennensis Gmelin, 1789 (c), Caprimulgus nigrescens Cabanis, 1848 (a), Hydropsalis climacocerca schomburgki P.L. Sclater, 1866 (b). Trochilidae: Glaucis hirsutus hirsutus (Gmelin, 1788) (a), Phaethornis malaris insolitus ${ }^{19}$ Zimmer, 1950 (b, 30363-65; 31655; 17272; 42466-67), Phaethornis bourcieri bourcieri2 (Lesson, 1832) (c, 50529-30), Phaethornis ruber nigricinctus Lawrence, 1858 (d, 50531), Campylopterus largipennis largipennis (Boddaert, 1783) (c, 34087), Florisuga mellivora mellivora (Linnaeus, 1758) (a, 50534-35), Anthracothorax nigricollis ${ }^{21}$ (Vieillot, 1817) (a), Chrysolampis mosquitus (Linnaeus, 1758) (a), Thalurania furcata nigrofasciata (Gould, 1861) (b, 17270-71; 42480; 45505; 43649), Hylocharis sapphirina (Gmelin, 1788) (a, 50532), Hylocharis cyanus viridiventris Berlepsch, 1880 (a, 50533), Polytmus theresiae leuchorous P.L. Sclater \& Salvin, 1867 (e, 54458; 34092$)$, Amazilia versicolor milleri (Bourcier, 1847) (a, 34095-96; 17275), Amazilia fimbriata fimbriata (Gmelin, 1788) (c, 42481; 27859), Heliodoxa aurescens (Gould, 1846) (a, 17277), Topaza pyra pyra22 (Gould, 1846) (b), Heliothryx auritus auritus (Gmelin, 1788) (c, 34098), Heliomaster longirostris longirostris (Audebert \& Vieillot, 1801) (a). Trogonidae: Pharomachrus pavoninus pavoninus (Spix, 1824) (a), Trogon melanurus eumorphus Zimmer, 1948 (f, 30234; 33315; 43018; 42482-83), Trogon viridis viridis Linnaeus, 1766 (a), Trogon rufus sulphureus Spix, 1824 (d, 43484), Trogon curucui peruvianus Swainson, 1937 (a), Trogon violaceus ramonianus Deville \& Dês Murs, 1849 (d). Alcedinidae: Ceryle torquatus torquatus (Linnaeus, 1766) (a), Chloroceryle amazona amazona (Latham, 1790) (a), Chloroceryle americana americana (Gmelin, 1788) (a), Chloroceryle inda inda (Linnaeus, 1766) (a), Chloroceryle aenea aenea (Pallas, 1764) $(a, 27865 ; 43025 ; 50536)$. Momotidae: Momotus momota microstephanus Sclater, 1858 (e, 29955-6; 43651; 42491; 17548; 31667).Galbulidae: Galbula albirostris chalcocephala Deville, 1849 (e, 42492-93; 52974-78), Galbula galbula (Linnaeus, 1766) (a, 43819-20), Galbula leucogastra23 Vieillot, 1817 (a, 27854-55; 52985), Galbula dea brunneiceps Todd, 1943 (a, 50537; 30035), Jacamerops aureus aureus (Statius Muller, 1776) (c). Bucconidae: Notharchus macrorhynchus hyperrhynchus Sclater, 1855 (a, 42495), Notharchus ordii (Cassin, 1851) (a), Notharchus tectus tectus (Boddaert, 1783) (a, 17546; 52988), Bucco tamatia tamatia Gmelin, 1788 (c, 27856-58), Bucco capensis Linnaeus, 1766 (a, 29047; 42496), Malacoptila fusca24 (Gmelin, 1788) (c, 50538-40; 52995), Micromonacha lanceolata ${ }^{25}$ (Deville, 1849) (f), Nonnula amaurocephala Chapman, 1921 (g), Monasa nigrifrons nigrifons (Spix, 1824) (a, 43821), Monasa morphoeus peruana Sclater, 1855 (d,

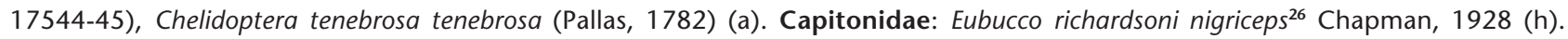
Ramphastidae: Pteroglossus castanotis castanotis Gould, 1834 (d, 42501), Pteroglossus pluricinctus Gould, 1835 (e), Pteroglossus azara azara $^{27}$ (Vieillot, 1819) (g, 42507), Selenidera nattereri (Gould, 1836) (b, 52998), Ramphastos vitellinus culminatus Gould, 1833 (c, 50544), Ramphastos tucanus cuvieri Wagler, 1827 (d). Picidae: Picumnus lafresnayi pusillus ${ }^{28}$ Pinto, 1936 (g), Colaptes punctigula guttatus ${ }^{29}$ Spix, 1824 (d, 27868), Piculus flavigula magnus (Cherrie \& Reichenberger, 1921) (a, 42508; 43823; 43704), Piculus chrysochloros capistratus (Malherbe, 1861) (e, 50545; 41591), Celeus elegans jumanus (Spix, 1824) (a), Celeus grammicus grammicus (Natterer \& Malherbe, 1845) (e, 17505; 42510), Celeus flavus peruvianus (Cory, 1919) (d), Celeus torquatus occidentalis (Hargitt, 1889) (d), Dryocopus

Revista Brasileira de Zoologia 24 (4): 919-940, dezembro 2007 
lineatus lineatus (Linnaeus, 1766) (a), Melanerpes cruentatus ${ }^{30}$ (Boddaert, 1783) (a), Veniliornis affinis orenocensis Berlepsch \& Hartert, 1902 (b, 17501-02), Campephilus melanoleucos melanoleucus (Gmelin, 1788) (a), Campephilus rubricollis rubricollis (Boddaert, 1783) (c, 42512; 29063). Furnariidae/Furnariinae: Synallaxis rutilans confinis Zimmer, 1935 (g), Cranioleuca vulpina vulpina ${ }^{31}$ (Pelzeln, 1856) (a), Xenops milleri (Chapman, 1914) (a, 50577; 54946; 17746), Xenops minutus remoratus Zimmer, 1935 (e, 50574-76; 45522; 53049), Berlepschia rikeri (Ridgway, 1886) (a, 53051-52), Hyloctistes subulatus subulatus (Spix, 1824) (a, 42573-74; 50578-79), Ancistrops strigillatus strigillatus (Spix, 1825) (a, 42575-76), Philydor pyrrhodes (Cabanis, 1848) (a, 43170; 42577; 43783; 53040), Automolus infuscatus badius Zimmer, 1935 (b, 42587-89; 50580-81; 53043-44; 42520), Automolus ochrolaemus turdinus (Pelzeln, 1859) (c, 53041-42), Automolus rubiginosus venezuelanus Zimmer \& Phelps, 1947 (b), Sclerurus rufigularis brunescens ${ }^{32}$ Todd, 1948 (g, 50582-83; 53048; 30429; 31690), Sclerurus caudacutus brunneus Sclater, 1857 (f, 42590-92). Furnariidae/Dendrocolaptinae: Dendrocincla fuliginosa neglecta ${ }^{33}$ Todd, 1948 (f, 50566-67; 42516; 43084), Dendrocincla merula bartleti Chubb, 1919 (d, 42521-22; 50568-69), Deconychura longicauda connectens Zimmer, 1929 (e), Deconychura stictolaema secunda Hellmayr, 1904 (d, 50558-61; 43825), Glyphorynchus spirurus rufigularis Zimmer, 1934 (c, 53015-16; 50562-63), Sittasomus griseicapillus amazonus Lafresnaye, 1850 (d, 28302-03; 30414), Nasica longirostris (Vieillot, 1818) (a), Dendrexetastes rufigula devillei (Lafresnaye, 1850) (f, 43099-10; 53020-21), Hylexestastes stresemanni streseman ${ }^{34}$ Snethlage, 1925 (g, 50548), Dendrocolaptes certhia radiolatus ${ }^{35}$ Sclater \& Salvin, 1868 (e, 42555; 49900; 43102; 50549), Dendrocolaptes picumnus validus $^{36}$ (Tschudi, 1844) (d, 43105-06), Xiphocolaptes promeropirhynchus orenocensis Berlepsch \& Hartert, 1902 (d), Xiphorhynchus picus picus (Gmelin, 1788) (a, 53025; 53027; 43111; 42556-57), Xiphorhynchus kienerii37 (Des Murs, 1855) (i, 43828; 43116), Xiphorhynchus obsoletus notatus Eyton, 1852 (e, 43120-21; 42558; 43128), Xiphorhynchus guttatoides ${ }^{38}$ (Lafresnaye, 1850) (d, 43139-41; 42570-71), Lepidocolaptes albolineatus duidae Zimmer, 1934 (b). Thamnophilidae: Cymbilaimus lineatus intermedius (Hartert \& Goodson, 1917) (a, 50584-85; 42593; 30044), Frederickena unduligera unduligera (Pelzeln, 1868) (b), Taraba major semifasciata (Cabanis, 1872) (a), Sakesphorus canadensis loretoyacuensis Bartlett, 1882) (a, 28297; 28292-93; 43833-34), Thamnophilus nigrocinereus cinereoniger Pelzeln, 1868 (b, 43834-35), Thamnophilus aethiops polionotus Pelzeln, 1868 (b, 50589; 5862), Thamnophilus schistaceus heterogynus (Hellmayr, 1907) (f, 42597-98; 42603; 43177; 43180), Thamnophilus murinus murinus Sclater \& Salvin, 1868 (a, 30435; 17344-48; 53059; 43673), Thamnophilus amazonicus cinereiceps Pelzeln, 1868 (b, 53064-65; 28289-90; 49967-68), Megastictus margaritatus (Sclater, 1855) (a, 53068-69), Pygiptila stellaris occipitalis Zimmer, 1932 (b, 43225; 42610; 43186), Thamnomanes ardesiacus obidensis (Snethlage, 1914) (c, 42611-13; 42636; 42633), Thamnomanes caesius glaucus (Cabanis, 1847 (c, 50587-88; 53073), Herpsilochmus dorsimaculatus Pelzeln, 1868 (c, 53110, 53112), Myrmotherula brachyura brachyura (Hermann, 1783) (a, 50601; 53075-76), Myrmotherula ambigua Zimmer, 1932 (b, 53081), Myrmotherula multostriata39 Sclater, 1858 (d), Myrmotherula cherriei40 Berlepsch \& Hartert, 1902 (e, 50595-98), Myrmotherula klagesi Todd, 1927 (i), Myrmotherula haematonota pyrrhonota Sclater \& Salvin, 1857 (c, 50599-00; 42644-45), Myrmotherula

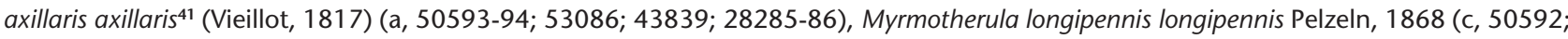
43840; 53096; 53094), Myrmotherula menetriesii pallida Berlepsch \& Hartert, 1902 (e, 53101; 43841; 53103), Myrmotherula assimilis assimilis Pelzeln, 1868 (a, 43842; 43188-89; 50025), Terenura spodioptila signata Zimmer, 1932 (b), Formicivora grisea rufiventris ${ }^{42}$ Carriker, 1936 (b, 34162-63), Cercomacra cinerascens cinerascens (Sclater, 1857) (b, 17356), Cercomacra tyrannina tyrannina ${ }^{43}$ (Sclater, 1855) (b, 52530-31; 52536; 50586; 43190), Myrmoborus lugubris stictopterus Todd, 1927 ( $\mathrm{g}, 43843-44)$, Myrmoborus myotherinus ardesiacus ${ }^{44}$ Todd, 1927 (g, 50616; 50620; 50618; 42660; 42668; 17342), Dichrozona cincta cincta (Sclater \& Salvin, 1880) (b), Hylophylax naevius consobrina Todd, 1913 (b, 50602-03; 43851; 42728; 42733), Hylophylax punctulatus punctulatus (Des Murs, 1856) (a, 46023; 50008), Hylophylax poecilinotus duidae ${ }^{45}$ Chapman \& Salvin, 1880 (b, 50611-13; 43183; 42738), Hypocnemis cantator saturata Carriker, 1930 (e, 17345; 53115; 30436), Hypocnemis hypoxantha hypoxantha Sclater, 1869 (d, 42677-80; 50604; 50605-07), Hypocnemoides melanopogon occidentalis Zimmer, 1932 (e, 50623; 43222), Sclateria naevia argentata (dês Murs, 1856) (d, 43226; 50013), Percnostola minor minor ${ }^{46}$ Pelzeln, 1868 (b, 43846-48; 45536-37; 53117), Schistocichla leucostigma infuscata ${ }^{47}$ (Todd, 1927) (e, 50590-91; 5311922; 42691), Myrmeciza disjuncta ${ }^{48}$ Friedmann, 1945 (b), Pithys albifrons brevibarba Chapman, 1928 (e, 50624-25; 42693-96; 3042326), Gymnopithys leucaspis laterallis Todd, 1927 (b, 50627-29; 42707-709), Rhegmatorhina cristata (Pelzeln, 1868) (b, 42724-25), Phlegopsis erythroptera erythroptera (Gould, 1855) (e, 42742-43; 50635-38). Formicariidae: Formicarius analis zamorae Chapman, 1923 (h, 42744), Formicarius colma colma Boddaert, 1783 (c, 50641; 30430; 42746), Grallaria varia cinereiceps Hellmayr, 1903 (b), Myrmothera campanisona dissors Zimmer, 1934 (b, 37523), Hylopezus macularius diversus (Zimmer, 1934) (e, 42749-50). Conopophagidae: Conopophaga aurita inexpectata Zimmer, 1931 (b, 42755; 42752; 45547). Tyrannidae/Tyranninae: Elaenia ruficeps ${ }^{49}$ Pelzeln, 1868 (a, 34284-65; 34281), Myiopagis gaimardii guianensis (Berlepsch, 1907) (a), Myiopagis flavivertex (Sclater, 1887) (a), Myiopagis caniceps cinerea (Pelzeln, 1868) (e, 50675-76; 53240; 17357; 17299), Phaeomyias murina wagae (Taczanowski, 1877) (a), Camptostoma obsoletum napaeum (Ridgway, 1888) (a, 43863), Tyrannulus elatus (Latham, 1790) (a), Ornithion inerme Hartlaub, 1853 (a), Zimmerius gracilipes gracilipes (Sclater \& Salvin, 1868) (e, 17306-07; 17312), Inezia subflava subflava ${ }^{50,51}$ (Sclater \& Salvin, 1873) $(\mathrm{a}, 28777 ; 28282)$, Mionectes oleagineus chloronotus (Lafresnaye \& d'Orbigny, 1837) (a, 50677; 42889; 42882-3; 34299), Mionectes macconnelli amazonus Tood, 1921 (a), Myiornis ecaudatus ecaudatus (d'Orbigny \& Lafresnaye, 1837) (a), Hemitriccus zosterops zosterops ${ }^{52}$ (Pelzeln, 1868) (d), Hemitriccus minor pallens ${ }^{53}$ (Todd, 1925) (f), Todirostrum maculatum anectans J. T. Zimmer, 1940 (i), Todirostrum chrysocrotaphum guttatum Pelzeln, 1868 (d, 53195), Corythopis torquatus sarayacuensis Chubb, 1818 (d, 50644-45; 42891; 42897; 45548), Platyrinchus platyrhynchos

Revista Brasileira de Zoologia 24 (4): 919-940, dezembro 2007 
platyrhynchos (Gmelin, 1788) (c, 53166-67; 30384), Platyrinchus coronatus coronatus Sclater, 1858 (a, 53168-69; 42873-75), Tolmomyias poliocephalus poliocephalus (Taczanowski, 1884) (d, 53186-87; 17298; 17300; 42877), Tolmomyias assimilis neglectus J. T. Zimmer, 1939 (b, 53180; 53176; 53178), Ramphotrigon ruficauda (Spix, 1825) (a, 50659-60; 39185; 46146; 50114-15), Cnipodectes subbrunneus minor P. L. Sclater, 1884 (d, 50661-63; 42876; 50067-68), Onychorhynchus coronatus castenaui Deville, 1849 (d, 50105-06), Myiobius barbatus amazonicus Todd, 1925 (d, 53164-5; 50664-67), Terenotriccus erythrurus venezuelensis ). t. zimmer, 1939 (b, 42856-57; 5066869; 28294; 53163; 30385; 45589), Lathrotriccus euleri bolivianus (J. A. Allen, 1889) (a, 53162; 42855; 43308), Cnemotriccus fuscatus duidae J. T. Zimmer, 1938 (b, 54459; 50073; 31730), Knipolegus poecilocercus (Pelzeln, 1868) (a, 28269; 28270; 28273), Attila citriniventris Sclater, 1859 (d, 48389), Attila cinnamomeus (Gmelin, 1789) (a, 42850), Attila spadiceus spadiceus (Gmelin, 1789) (a), Rhytipterna simplex frederici (Bangs \& T. E. Penard, 1918) (a, 50567), Rhytipterna immunda (Sclater \& Salvin, 1873) (a, 34242; 32241), Myiarchus ferox ferox (Gmelin, 1789) (a), Myiarchus tuberculifer tuberculifer (d'Orbigny \& Lafresnaye, 1837) (a), Megarynchus pitangua pitanga (Linnaeus, 1766) (a, 30378; 50092, 39177), Pitangus sulphuratus sulphuratus (Linnaeus, 1766) (a), Philohydor lictor lictor (Lichtenstein, 1823) (a), Myiozetetes cayanensis cayennensis (Linnaeus, 1766) (a, 17318; 53158), Myiozetetes similis similis (Spix, 1825) (a), Conopias trivirgatus berlepschi E. Snethlage, 1914 (a, 50098; 37526; 12152), Conopias parvus parvus (Pelzeln, 1868) (c), Legatus leucophaius leucophaius (Vieillot, 1818) (a), Empidonomus varius rufinnus (Spix, 1825) (a, 34236; 17330-34), Tyrannopsis sulphurea (Spix, 1825) (a), Tyrannus melancholicus melancholicus Vieillot, 1819 (a, 17326; 30054; 28263; 30053; 30379), Pachyramphus surinamus (Linnaeus, 1766) (a), Pachyramphus polychopterus tristis (Kaup, 1852) (a), Pachyramphus marginatus nanus Bangs \& T. E. Penard, 1921 (a, $43234-$ 35), Pachyramphus rufus rufus (Boddaert, 1783) (a), Pachyramphus castaneus saturatus Chapman, 1914 (d), Tityra inquisitor albitorques Du Bus de Gisignies, 1847 (a), Tityra cayana cayana (Linnaeus, 1766) (a, 30098-100; 28325). Tyrannidae/Piprinae: Schiffornis turdina amazona (P. L. Sclater, 1861) (a, 50646-50; 45557-58; 37031), Schiffornis major major ${ }^{54}$ Des Murs, 1856 (a, $\left.43860 ; 43299\right)$, Tyranneutes stolzmanni (Hellmayr, 1906) (a, 53144; 50053), Neopelma chrysocephalum (Pelzeln, 1868) (c, 50658; 53151-52; 34228; 31725-6), Neopipo cinnamomea cinnamomea (Lawrence, 1869) (a, 43694), Piprites chloris tschudii (Cabanis, 1874) (e, 53153), Heterocercus flavivertex Pelzeln, 1868 (c, 28326-7), Xenopipo atronitens Cabanis, 1847 (a), Chiroxiphia pareola regina P. L. Sclater, 1856 (a, $43298 ; 50050$; 42849), Lepidothrix coronata carbonata (Todd, 1925) (e, 50654-56; 42776-77; 42807), Dixiphia pipra pipra (Linnaeus, 1758$)$ (c, 5065153; 53140), Pipra erythrocephala erythrocephala ${ }^{55}$ (Linnaeus, 1758) (a, 42761; 42768; 42770; 45550), Pipra filicauda filicauda Spix, 1825 (a, 42830-31; 43277-78; 43855-56). Tyrannidae/Cotinginae: Cotinga cayana (Linnaeus, 1766) (a, 30086-88), Xipholena punicea (Pallas, 1764) (a, 30112; 83; 402), Laniocera hypopyrra (Vieillot, 1817) (a, 43237), Lipaugus vociferans (Wied, 1820) (a, 45540; 43852; 42758; 30506), Gymnoderus foetidus (Linnaeus, 1758) (a), Perissocephalus tricolor (Statius Muller, 1776) (c), Phoenicircus nigricollis (Linnaeus, 1758) (c). Troglodytidae: Thryothorus coraya griseipectus Sharpe, 1881 (e, 42899; 50682; 53245; 45565), Thryothorus leucotis albipectus Cabanis, 1849 (a, 42900), Troglodytes musculus albicans Berlepsch \& Taczanowski, 1883 (a, 54460; 23742; 31262), Cyphorhinus arada transfluviatilis Todd, 1932 (e, 42912-11; 42907; 42916-17; 30241; 30238), Microcerculus bambla albigularis ${ }^{56}$ (Sclater, 1858) (h, 5324648; 4556; 42901-05). Sylviidae: Microbates collaris collaris (Pelzeln, 1868) (a, 50680-81; 53252-53), Ramphocaenus melanurus duidae Zimmer, JT, 1937 (b, 53258-59), Polioptila plumbea plumbea (Gmelin, 1788) (a, 28323-24), Polioptila guianensis facilis Zimmer, 1942 (b, 50678-79; 53260). Turdidae: Turdus leucomelas ephippialis Sclater, 1862 (a), Turdus albicollis phaeopygys Cabanis, 1848 (c, $42922-25$; 30375-77; 53250), Turdus hauxwelli57 Lawrence, 1869 (d), Turdus fumigatus fumigatus Lichtenstein, 1823 (a, 28310; 43870). Vireonidae: Cyclarhis gujanensis gujanensis (Gmelin, 1789) (a), Vireolanius leucotis leucotis (Swainson, 1838) (c), Vireo olivaceus olivaceus (Linnaeus, 1766) (a, 17566; 17573; 17567; 17569), Hylophilus semicinereus viridiceps (Todd, 1929) (c, 42929-30), Hylophilus thoracicus griseiventris Berlepsch \& Hartert, 1902 (a, 298), Hylophilus hypoxanthus hypoxanthus ${ }^{58}$ Pelzeln, 1868 (e, 50683; 33831; 47950), Hylophilus brunneiceps ${ }^{59}$ Sclater, 1866 (b, 53284), Hylophilus ochraceiceps ferrugineifrons Sclater, 1862 (b, 53281-83-80-79. Emberezidae/Coerebinae: Coereba flaveola minima (Bonaparte, 1854) (c, 28221-22; 34316-17). Emberezidae/Thraupinae: Dacnis cayana cayana (Linnaeus, 1766) (a, 28320; 30362; 17443), Dacnis flaviventer d'Orbigny \& Lafresnaye, 1837 (a, 42933; 17446), Cyanerpes nitidus (Hartlaub, 1847) (a), Cyanerpes caeruleus microrhyncha Berlespch, 1884 (d, 34319; 50686; 17438), Cyanerpes cyaneus dispar Zimmer, 1942 (b), Chlorophanes spiza caerulescens Cassin, 1864 (d), Hemithraupis guira nigrigula (Boddaert, 1783) (a), Hemithraupis flavicollis aurigularis Cherrie, 1916 (b, 53264; 17401), Tangara cayana cayana (Linnaeus, 1766) (a, 27883), Tangara punctata punctata (Linnaeus, 1766) (a), Tangara mexicana boliviana (Bonaparte, 1851) (d), Tangara velia iridina (Hartlaub, 1841) (a, 30368-69), Tangara chilensis coelicolor (Sclater, 1851) (b, 50687), Euphonia chlorotica amazonica Parkes, 1969 (f), Euphonia rufiventris (Vieillot, 1819) (a), Euphonia plumbea Du Bus, 1855 (c), Euphonia chrysopasta chrysopasta Sclater \& Salvin, 1869 (d). Emberezidae/Catamblyrhynchinae: Thraupis palmarum melanoptera (Sclater, 1857) (a, 30084; 42935; 17388), Ramphocelus carbo carbo (Pallas, 1764) (a, 27879-78; 30343-45), Ramphocelus nigrogularis (Spix, 1825) (a, 42937), Habia rubica peruviana (Taczanowski, 1884) (a, 42939-41), Eucometis penicillata penicillata (Spix, 1825) (a, 43872-3), Lanio fulvus peruvianus Carriker, 1934 (h, 42945-44), Tachyphonus luctuosus luctuosus d'Orbigny \& Lafresnaye, 1837 (a), Tachyphonus cristatus cristatellus Sclater, 1862 (c, 50684-85; 30075; 17390-93), Tachyphonus surinamus brevipes Lafresnaye, 1846 (e, 17389), Tachyphonus phoenicius Swainson, 1838 (a, 54461; 34345), Schistochlamys melanopis aterrima Todd, 1912 (b), Cissopis leverianus leverianus (Gmelin, 1788) (A). Emberezidae/Icterinae: Sturnella militaris militaris (Linnaeus, 1758) (a), Lampropsar tanagrinus tanagrinus (Spix, 1824) (a, 42931-32), Molothrus oryzivorus oryzivorus (Gmelin, 1788) (a), Icterus chrysocephalus (Linnaeus, 1766) (c,

Revista Brasileira de Zoologia 24 (4): 919-940, dezembro 2007 
17394, 18785), Cacicus haemorrhous haemorhous (Linnaeus, 1766) (a), Cacicus cela cela (Linnaeus, 1758) (a, 28308-9), Psarocolius decumanus decumanus (Pallas, 1769) (c). Emberezidae/Cardinalinae: Saltator grossus grossus (Linnaeus, 1766) (a, 17404), Caryothraustes canadensis canadensis (Linnaeus, 1766) (c, 50688; 30057), Saltator maximus maximus (Statius Muller, 1776) (a, 17402-03), Paroaria gularis gularis (Linnaeus, 1766) (a, 28313), Cyanocompsa cyanoides rothschildii (bartlett, 1890) (a, 17406; 30373; 43875). Emberezidae/ Emberezinae: Volatinia jacarina splendens (Vieillot, 1817) (a, 34360; 17411), Dolospingus fringilloides (Pelzeln, 1870) (b), Sporophila americana americana (Gmelin, 1789) (a), Sporophila nigricollis nigricollis (Vieillot, 1823) (a), Sporophila castaneiventris Cabanis, 1849 (a), Sporophila angolensis torridus (Scopoli, 1769) (a, 17418; 17420; 17419), Arremon taciturnus taciturnus (Hermann, 1783) (a, 17424), Ammodramus aurifrons aurifrons $^{60}$ (Spix, 1825) (a, 17332-37), Emberizoides herbicola sphenurus ${ }^{61}$ (Vieillot, 1818) (i), Sicalis columbiana goeldii Berlepsch, 1906 (a).

\section{Notas taxonômicas e biogeográficas}

1 Taxonomia segundo del Hoyo et al. (1992), ${ }^{2}$ Taxonomia segundo del Hoyo et al. (1992), ${ }^{3}$ Taxonomia segundo deL Hoyo et al. (1994),

${ }^{4}$ Taxonomia segundo del Horo et al. (1994), ${ }^{5}$ Taxonomia segundo Del Hoyo et al. (1994), 6 Taxonomia segundo DeL Horo et al. (1994),

7 Taxonomia segundo del Hoyo et al. (1994), ${ }^{8}$ Taxonomia segundo del Horo et al. (1994), ${ }^{9}$ Aparentemente substituído no alto Rio Negro por O. g. medius (Friedmann 1948), ${ }^{10}$ Taxonomia segundo Haffer (1974), 11 Taxonomia segundo del Hoyo et al. (1997), 12 Taxonomia segundo DEL Horo et al. (1997), ${ }^{13}$ Taxonomia segundo DeL Horo et al. (1997), ${ }^{14}$ Taxonomia segundo deL HoYo et al. (1997), 15 Para distribuição e ecologia desta espécie ver MartusCell \& Yamashita (1997), 16 Taxonomia segundo del Hoyo et al. (1997), 17 Taxonomia segundo DEL Horo et al. (1999), 18 Taxonomia e distribuição geográfica segundo RoBıNS \& PARKER (1997), 19 Taxonomia e distribuição geográfica segundo HinkelmANn (1996), ${ }^{20}$ Aparentemente substituído no alto Rio Negro por P. b. whitelyi (FriedmANN 1948), ${ }^{21}$ Taxonomia segundo DEL Horo et al.(1999), ${ }^{22}$ Taxonomia e distribuição geográfica seguem Hu et al. (2000), ${ }^{23}$ Taxonomia e distribuição geográfica segundo Del Horo et al. (2002), ${ }^{24}$ Taxonomia e distribuição geográfica segundo Del Horo et al. (2002), ${ }^{25}$ Taxonomia e distribuição geográfica seguem DeL HoYo et al. (2002), ${ }^{26}$ Taxonomia e distribuição geográfica seguem Del HoYo et al. (2002), 27 Taxonomia e distribuição geográfica seguem DEL HoYo et al. (2002), ${ }^{28}$ Taxonomia e distribuição geográfica seguem DEL HoYo et al. (2002), ${ }^{29}$ Taxonomia e distribuição geográfica seguem DEL HoYo et al. (2002), ${ }^{30}$ Taxonomia e distribuição geográfica seguem DEL HoYO et al. (2002), ${ }^{31}$ ZIMMER (1997) recomenda tratar alopecias sinônimo de vulpina tratamento adotado aqui, ${ }^{32}$ Aparentemente substituído no alto Rio Negro por S. r. fulvigularis (Friedmann 1948), ${ }^{33}$ Aparentemente substituído no alto Rio Negro por D. f. phaeochroa (FriedmanN 1948), ${ }^{34}$ Aparentemente substituído no alto Rio Negro por H. s. insignis (FrIEDMANn 1948), ${ }^{35}$ Distribuição geográfica segundo Marantz (1997), ${ }^{36}$ distribuição geográfica segundo Marantz (1997), ${ }^{37}$ Taxonomia proposta por Aleixo \& Whitney (2002), 38 Taxonomia proposta por Aleixo (2002), 39 Taxonomia e distribuição geográfica propostas por IsLER et al. (1999), ${ }^{40}$ Distribuição geográfica propostas por IsLER et al. (1999), 41 Substituída por M. a. melaena no alto Rio Negro e setores ao norte do Rio Branco e região do Solimões (Tefé) (FrIEDMANN 1948, PINTO 1978), ${ }^{42}$ Para distribuição geográfica desta subespécie consultar DEL HoYo et al. (2003), ${ }^{43}$ Distribuição geográfica segundo BieRREGAARD et al. (1997), ${ }^{44}$ A forma do alto Rio Negro e Roraima é elegans com o ventre mais clara. Material do Jaú e Maraã é de ardesiacus com ventre mais cinza (Haffer \& FitZPATRICK 1985), 45 Ver nota taxonômica em Ridgely \& TUdor (1994), ${ }^{46}$ Taxonomia e distribuição geográfica seguem Capparella et al. (1997), ${ }^{47}$ Tratado por Pinto (1978) como Sclateria leucostigma infuscata, taxonomia segue RidGely \& TUdor (1994), ${ }^{48}$ Para revisão da ecologia e distribuição geográfica da espécie consultar Zimmer (1999), Borges \& AlmeidA (2001) e NAKA et al. (2006), 49 Distribuição geográfica segundo deL HoYo et al. (2004), ${ }^{50}$ Nomenclatura proposta por ZIMMER \& WHITTAKER (2000), ${ }^{51}$ SUbstituído por I. s. obscura no alto Rio Negro (ZIMmer \& WHITTAKER 2000), ${ }^{52}$ Taxonomia e distribuição geográfica segundo deL Hoyo et al. (2004), ${ }^{53}$ Taxonomia e distribuição geográfica segundo DEL HoYo et al. (2004), ${ }^{54}$ Aparentemente substituído no noroeste da Amazônia por S. m. duidae (Friedmann 1948), ${ }^{55}$ Aparentemente substituído por P. e. berlespshi no alto Rio Negro (FriedmanN 1948), ${ }^{56}$ Aparentemente substituído por M. c. caurensis no alto Rio Negro (Friedmann 1948), ${ }^{57}$ Recomendações taxonômicas seguem Ridgely \& Tudor (1994), ${ }^{58}$ Para considerações taxonômicas consultar Zimmer et al. (1997), HAfFer (1997) e RidGely \& Tudor (1994), ${ }^{59}$ Taxonomia segue RidGely \& Tudor (1994), ${ }^{60}$ Aparentemente substituído por A. aurifrons tenebrosus no alto Rio Negro (FriedmanN 1948), ${ }^{61}$ Taxonomia segundo EISENMANN \& SHORT (1982).

Revista Brasileira de Zoologia 24 (4): 919-940, dezembro 2007 